\title{
THE TRANSFORMATION OF WIENER INTEGRALS BY NONLINEAR TRANSFORMATIONS
}

\author{
BY
}

\author{
R. H. CAMERON AND W. T. MARTIN
}

Introduction. In this paper we study the behavior of Wiener integrals under transformations of the form

$$
T: \quad y(t)=x(t)+\Lambda(x \mid t),
$$

where $\Lambda(x \mid t)$ is a functional depending on the function $x$ and the number $t$ and satisfying certain smoothness conditions. (The number $t$ ranges over the interval $0 \leqq t \leqq 1$, and the function $x$ ranges over a measurable subset $\Gamma$ of the space $C$ of continuous functions on $0 \leqq t \leqq 1$ which vanish when $t=0$.) The smoothness conditions on $\Lambda(x \mid t)$ require in particular that it have a Volterra derivative $K(x \mid t, s)$ such that

$$
\left.\delta \Lambda \equiv \frac{\partial}{\partial h} \Lambda(x+h \delta x \mid t)\right]_{h=0}=\int_{0}^{1} K(x \mid t, s) \delta x(s) d s .
$$

This Volterra derivative is somewhat analogous to the matrix of the partial derivatives of the $n$ functions which define a transformation in $n$ dimensions (or, more strictly, to this matrix minus the identity matrix), and hence it is natural to think of the Fredholm determinant $D(x)$ of $K(x \mid t, s)$,

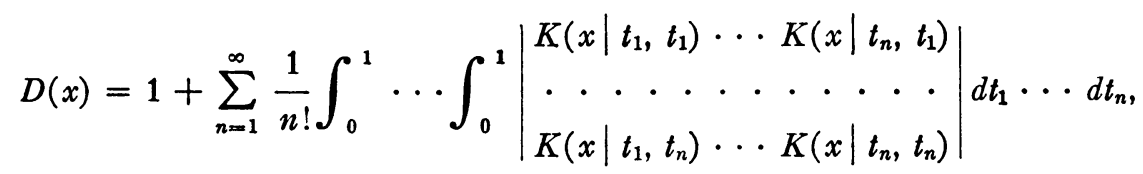

as being analogous to the functional determinant or Jacobian of an $n$-dimensional transformation. Consequently it is reasonable to think of $D(x)$ as the "volume element ratio" of $y$ and $x$, and write

$$
\frac{d V_{y}}{d V_{x}}=D(x) .
$$

If we wished to define the "volume elements" separately, we might perhaps choose $d V_{x}$ arbitrarily and then define $d V_{y}$ by (0.3). However, there are certain reasons why it is preferable to think of the volume element as being almost always infinite, and working instead with the "measure element" which is finite because of the exponentials which occur in the definition of

Presented to the Society, September 7, 1948; received by the editors April 5, 1948. 
the Wiener integral $[5]\left({ }^{1}\right)$. Though these exponentials were suggested originally by probability theory, we shall regard them from a purely analytical point of view and consider them to be convergence factors. The heuristic relationship between the "volume element" and "measure element" is suggested by Wiener's definition of a quasi-interval ard its measure. The set $Q$ of elements $x \in C$ satisfying the following inequalities is called a quasiinterval:

$Q: \alpha_{j}<x\left(t_{j}\right)<\alpha_{j}+\Delta \alpha_{j} ; j=1, \cdots, n$ (where $0=t_{0}<t_{1}<\cdots<t_{n} \leqq 1$ ); and the measure of $Q$ is defined by the integral

$$
\begin{aligned}
m_{w}(Q)= & \frac{1}{\left(\pi^{n} \Delta t_{1} \cdot \Delta t_{2} \cdots \Delta t_{n}\right)^{1 / 2}} \int_{\alpha_{n}}^{\alpha_{n}+\Delta \alpha_{n}} \cdots \int_{\alpha_{1}}^{\alpha_{1}+\Delta \alpha_{1}} \\
& \cdot \exp \left(-\sum_{j=1}^{n} \frac{\left(\Delta \xi_{j}\right)^{2}}{\Delta t_{j}}\right) d \xi_{1} \cdots d \xi_{n}, \\
& \quad \text { where } \Delta t_{j}=t_{j}-t_{j-1} \text { and } \Delta \xi_{j}=\xi_{j}-\xi_{j-1}, \xi_{0}=0 .
\end{aligned}
$$

(Here $\Delta \xi_{j}$ is not really an increment, but a difference of two independent variables. In the limit, however, as the quasi-interval closes down on one function $x(t)$, these $\Delta \xi_{j}$ suggest $d x(t)$.) If we drop the factors before the integral and let $n \rightarrow \infty, \Delta t_{j} \rightarrow 0, \Delta \alpha_{j} \rightarrow 0$, some such relationship as the following between the measure and volume elements is suggested.

$$
d_{w} x=\exp \left(-\int_{0}^{1}\left[\frac{d x(t)}{d t}\right]^{2} d t\right) d V_{x}
$$

The dubious character of this "equation" is seen not only by the way it is introduced here, but also because the exponential factor exists only on a set of $x$ of measure zero in $C$. (The set of elements $x(t)$ which are absolutely continuous and hence are the integrals of their derivatives $x^{\prime}(t)$ is known to be a null set in $C$. Even if we attempt to interpret the integral in the Hellinger sense, it is still almost always infinite.) Nevertheless the formalism (0.4) is very suggestive, and we may take it as defining the "volume element" $d V_{x}$ in terms of the "measure element" $d_{w} x$, the volume element being infinite when the exponential factor fails to exist. The suggestive value of the formalism (0.4) is immediately seen if we write the corresponding equation for $y$

$$
d_{w} y=\exp \left(-\int_{0}^{1}\left[\frac{d y(t)}{d t}\right]^{2} d t\right) d V_{y}
$$

and then eliminate the "volume elements" $d V_{x}$ and $d V_{y}$ between (0.3), (0.4), (0.5); at the same time rewriting the formal integral which arises from the difference of the two exponents as a Riemann-Stieltjes integral:

(1) Numbers in brackets refer to the references cited at the end of the paper. 


$$
\frac{d_{w} y}{d_{w} x}=D(x) \exp \left(-\int_{0}^{1}\left\{\frac{d}{d t}[y(t)-x(t)]\right\} d[y(t)+x(t)]\right) .
$$

This formula (0.6) for the "measure element ratio" turns out to be meaningful for all $x$ in $C$ if we sufficiently restrict $\Lambda$; for the difference $y(t)-x(t)$ is merely $\Lambda(x \mid t)$, and we assume hypotheses which cause this to have a derivative of bounded variation. Thus the integrand $d[y(t)-x(t)] / d t$ is of B.V. and the integrator $y(t)+x(t)$ is continuous, and hence the integral exists in the strict Riemann-Stieltjes sense.

Not only does the right member of (0.6) turn out to exist for all $x \in C$, so that the ratio of $d_{w} y$ and $d_{w} x$ is everywhere defined and $d_{w} y$ is known if $d_{w} x$ is chosen arbitrarily, but it also turns out that the left member behaves as one would expect in connection with the transformation of integrals. In fact, we have

$$
\int_{T \Gamma}^{C} F(y) d_{w} y=\int_{\Gamma}^{C} F(y)\left|\frac{d_{w} y}{d_{w} x}\right| d_{w} x
$$

or

$$
\begin{aligned}
& \int_{T \Gamma}^{C} F(y) d_{w} y \\
& =\int_{\Gamma}^{C} F(T x)|D(x)| \exp \left(-\int_{0}^{1} \frac{d}{d t}[T x(t)-x(t)] d[T x(t)+x(t)]\right) d_{w} x
\end{aligned}
$$

It is the purpose of this paper to establish formula (0.8) (and of course at the same time the equivalent (0.7)) under suitable conditions on $\Lambda(x \mid t)$, and this is done in Theorems $I-V$ in the body of the paper. The last of these, Theorem V, is the final and most general theorem, and is the culmination to which the earlier ones lead.

In $\$ 1$ certain smoothness conditions on $\Lambda$ are introduced which imply the existence of the Volterra derivative $K$, and their properties are discussed, and in $\S \S 2,3$ the 1-to-1-ness of the transformation $T$ and of approximating transformations to it are studied. In $\$ 4$ an approximating " $n$-dimensional" form of (0.8) (namely (4.2)) is established in Theorem I, and in $\$ \$ 5-8$ certain lemmas are obtained which enable us to take a limit on (4.2) and obtain Theorem II in $\$ 9$. Theorem II establishes $(0.8)$ without any restriction to finite dimensionality, but with a restriction on the size of $K$, namely, $\left[\int_{0}^{1}[K(x \mid t, s)]^{2} d s\right]^{1 / 2} \leqq \lambda<1$. This restriction is removed in $\$ \S 10-14$ through the use of two local theorems III and IV. The final theorem establishing (0.8) "in the large" under general conditions is given in $\$ 14$, Theorem V. This is obtained by applying the local Theorem IV in a countable set of neighborhoods and then using the complete additivity of the Wiener integral to obtain the result "in the large." 
This paper is the first attack that the authors (or anyone else) have made upon the subject of nonlinear transformations of Wiener integrals. It does not, however, contain as special cases all of the results of our paper on linear transformations $[1, b]$ since the present paper puts stronger smoothness conditions on the kernel $K(x \mid t, s)$. In particular, it requires $K$ to be continuous in $t$ and $s$ for each $x$, while the $K(t, s)$ of $[1, \mathrm{~b}]$ is permitted to have a jump along the diagonal, $J(s)=K\left(s, s^{-}\right)-K\left(s, s^{+}\right)$. In the present paper the corresponding $J(x \mid s)$ is restricted to be identically zero. The authors hope to remove this restriction in a future paper, so that the integral transformations can have kernels of Volterra type as well as Fredholm type.

1. As stated in the Introduction we shall be concerned with transformations of the form

$$
T: \quad y(t)=x(t)+\Lambda(x \mid t) .
$$

The function $x$ is allowed to vary over some subset $S$ of the space $C$ which consists of all functions $x(t)$ defined and continuous on $0 \leqq t \leqq 1$ and vanishing at $t=0$. The variable $t$ is allowed to vary over the interval $I: 0 \leqq t \leqq 1$. Thus $\Lambda(x \mid t)$ is defined for $(x, t) \in S \otimes I$, the direct product of $S$ and $I$. (Similarly if we say $f(t, s)$ is defined for $(t, s) \in I^{2}$, or $(t, s) \in I \otimes I$, we shall mean that $f(t, s)$ is defined for all number pairs $(t, s)$ such that $(0 \leqq t \leqq 1,0 \leqq s \leqq 1))$. We let $\|x\|$ and $\|x\| \|$ denote the norms of $x$ in the Hilbert and uniform topologies respectively; that is, $\|x\|=\left\{\int_{0}^{1}[x(t)]^{2} d t\right\}^{1 / 2}$ and $\|x\|=\max _{t \in I}|x(t)|$. We say a subset $S$ of $C$ is convex if $x+v(y-x) \in S$ whenever $(x, y, v) \in S^{2} \otimes I$. We deal in this paper with functionals which are of "smooth variation" in the sense of the following definition:

Definition. A functional $\Lambda(x \mid t)$ defined on $S \otimes I$, where $S$ is a convex subset of $C$ open in the uniform topology, will be said to be "of smooth variation" if its first variation

$$
\left.\delta \Lambda(x|t| y) \equiv \frac{\partial}{\partial v} \Lambda(x+v y \mid t)\right]_{v=0}
$$

exists for all $(x, t, y)$ in $S \otimes I \otimes C$ and is representable in the form

$$
\delta \Lambda(x|t| y)=\int_{0}^{1} K(x \mid t, s) y(s) d s, \quad(x, t, y) \in S \otimes I \otimes C ;
$$

where $K(x \mid t, s)$ is continuous in $(x, t, s)$ throughout $S \otimes I^{2}$ (continuity in $x$ being understood in the uniform topology). The functional $K(x \mid t, s)$ will be called the kernel of the variation of $\Lambda$-it is the classical Volterra derivative of the functional $\Lambda$. (See [3, p. 25].)

We note at once that this hypothesis of continuity implies that for each $x$ in $S, K$ is continuous in $x$ uniformly with respect to all $(t, s)$ in $I^{2}$. This follows at once from the fact that we have a two-dimensional Heine-Borel 
theorem in $I^{2}$. The representation of $\Lambda$ in terms of $K$ is given in the following lemma.

LEMMA 1. Let $\Lambda(x \mid t)$ be a functional of smooth variation defined on $S \otimes I$, where $S$ is a (uniformly) open convex subset of $C$, and let $K(x \mid t, s)$ be the kernel of its variation. Then if $x_{0} \in S$, we have

(1.4) $\Lambda(x \mid t)=\Lambda\left(x_{0} \mid t\right)+\int_{0}^{1} d v \int_{0}^{1} K\left[x_{0}+\left(x-x_{0}\right) v \mid t, s\right]\left[x(s)-x_{0}(s)\right] d s$,

for all $(x, t) \in S \otimes I$. Moreover the kernel $K$ is completely defined in $S \otimes I^{2}$ by the functional $\Lambda$.

To see this, we note that if $y \in C$ and $x+v_{0} y \in S$ and $t \in I$, the variation $\delta \Lambda\left(x+v_{0} y|t| y\right)$ must exist and by (1.2),

$$
\begin{aligned}
\delta \Lambda\left(x+v_{0} y|t| y\right) & \left.=\frac{\partial}{\partial u} \Lambda\left(x+v_{0} y+u y \mid t\right)\right]_{u=0} \\
& \left.=\frac{\partial}{\partial v} \Lambda\left(x+v_{0} y+\left(v-v_{0}\right) y \mid t\right)\right]_{v=v_{0}} \\
& \left.=\frac{\partial}{\partial v} \Lambda(x+v y \mid t)\right]_{v=v_{0}}
\end{aligned}
$$

so that the last member of (1.5) must exist; and by (1.3),

$$
\left.\frac{\partial}{\partial v} \Lambda(x+v y \mid t)\right]_{v=v_{0}}=\int_{0}^{1} K\left(x+v_{0} y \mid t, s\right) y(s) d s
$$

when $\left(x+v_{0} y, t, y\right) \in S \otimes I \otimes C$. But since $S$ is convex, if $(x, x+y, v) \in S^{2} \otimes I$, $x+v y \in S$, and hence when $(x, x+y, v, t) \in S^{2} \otimes I^{2}$,

$$
\frac{\partial}{\partial v} \Lambda(x+v y \mid t)=\int_{0}^{1} K(x+v y \mid t, s) y(s) d s .
$$

Now for fixed $x, y, t$, the hypothesis on $K$ makes $K(x+v y \mid t, s)$ continuous in $(v, s)$ throughout $I^{2}$ so that the left member of $(1.7)$ is continuous in $v$, and we can apply the fundamental theorem of the integral calculus to obtain

$$
\Lambda\left(x+v_{1} y \mid t\right)-\Lambda\left(x+v_{0} y \mid t\right)=\int_{v_{0}}^{v_{1}} d v \int_{0}^{1} K(x+v y \mid t, s) y(s) d s
$$

when $\left(x, x+y, v_{0}, v_{1}, t\right) \in S^{2} \otimes I^{3}$; and when we substitute $x=x_{0}, y=x-x_{0}$, $v_{0}=0, v_{1}=1$, we obtain (1.4) for all $(x, t) \in S \otimes I$.

Finally, to show the uniqueness of $K$, we choose

$$
y_{u}(t)=\left\{\begin{array}{cl}
0, & 0 \leqq t \leqq u, \\
t-u, & u \leqq t \leqq 1,
\end{array} \quad(u, t) \in I^{2},\right.
$$


and note that $y_{u}(\cdot) \in C$ for all $u \in I$. Thus (1.3) must hold for $y=y_{u}$ so that when $(x, t, u) \in S \otimes I^{2}$

$$
\delta \Lambda\left(x|t| y_{u}\right)=\int_{0}^{1} K(x \mid t, s) y_{u}(s) d s=\int_{u}^{1} K(x \mid t, s)(s-u) d s .
$$

Thus when $(x, t, u) \in S \otimes I^{2}$,

$$
\left.K(x \mid t, u)=\frac{\partial^{2}}{\partial u^{2}}\left[\delta \Lambda\left(x|t| y_{u}\right)\right]=\frac{\partial^{2}}{\partial u^{2}}\left\{\frac{\partial}{\partial v} \Lambda\left(x+v y_{u} \mid t\right)\right]_{v=0}\right\},
$$

and $K$ is completely defined when $\Lambda$ is given.

COROLLARY 1. If $\Lambda$ is of smooth variation in $S \otimes I$ ( $S$ being a uniformly open convex subset of $C$ ) and $K$ is its kernel, and if $\Lambda\left(x_{0} \mid t\right)$ is a continuous function of $t$ on I for at least one $x_{0} \in S$, then $\Lambda(x \mid t)$ is continuous in $t$ on I for every fixed $x \in S$.

This follows at once from (1.4). Similarly we have the following corollaries.

CoROLlary 2. If $\Lambda$ is of smooth variation in $S \otimes I$ having kernel $K$, and $\Lambda\left(x_{0} \mid \cdot\right) \in C$ for some fixed $x_{0}$ in $S$ and $K(x \mid 0, s)=0$ for all $(x, s)$ in $S \otimes I$, then $\Lambda(x \mid \cdot) \in C$ for all $x$ in $S$.

COROLlaRY 3. If $\Lambda$ is of smooth variation in $S \otimes I$ and its kernel $K$ satisfies the inequality

$$
\left\{\int_{0}^{1}[K(x \mid t, s)]^{2} d s\right\}^{1 / 2} \leqq \lambda
$$

for all $(x, t)$ in $S \otimes I$, then $\Lambda$ satisfies the Lipschitz condition

$$
\||\Lambda(x \mid \cdot)-\Lambda(y \mid \cdot)|\| \leqq \lambda\|x-y\| \leqq \lambda\|x-y\|
$$

for all $(x, y)$ in $S^{2}$. This of course implies that $\Lambda(x \mid \cdot)$ is continuous in both the uniform and Hilbert topologies.

This follows immediately from (1.4) by means of the Schwarz inequality.

COROLlary 4. Let $\Lambda$ be of smooth variation in $S \otimes I$ and let its kernel $K(x \mid t, s)$ have a partial derivative $K_{t}(x \mid t, s)=\partial K(x \mid t, s) / \partial t$ which is continuous for all $(x, t, s)$ in $S \otimes I^{2}$ (uniform topology) and let $\Lambda_{t}\left(x_{0} \mid t\right)$ exist for all $t$ in $I$ and some one $x_{0}$ in $S$. Then $\Lambda_{t}(x \mid t)$ exists for all $(x, t)$ in $S \otimes I$ and $\Lambda_{t}(x \mid \cdot)$ is continuous in the uniform topology throughout $S$. In fact

$$
\begin{aligned}
\Lambda_{t}(x \mid t)= & \Lambda_{t}\left(x_{0} \mid t\right) \\
& +\int_{0}^{1} d v \int_{0}^{1} K_{t}\left[x_{0}+\left(x-x_{0}\right) v \mid t, s\right]\left[x(s)-x_{0}(s)\right] d s .
\end{aligned}
$$


Moreover if $\Lambda_{t}\left(x_{0} \cdot t\right)$ is continuous in $I, \Lambda_{t}(x \mid t)$ is continuous in $(x, t)$ throughout $S \otimes I$ (uniform topology) and is bounded in $S \otimes I$ if $K_{t}(x \mid t, s)$ is bounded in $S \otimes I^{2}$ and $\Lambda_{t}\left(x_{0} \mid t\right)$ is bounded for $t$ on $I$ and $S$ is bounded in the Hilbert topology. Finally, if $K_{t}(x \mid t, s)$ is of bounded variation in $t$ on $I$ for each $(x, s)$ in $S \otimes I$ and $\operatorname{Var}_{t \in I}\left[K_{t}(x \mid t, s)\right]$ is bounded in $(x, s)$ over $S \otimes I$ and $\Lambda_{t}\left(x_{0} \mid t\right)$ is of $B . V$. for $t$ on $I$ and $S$ is bounded in the Hilbert topology, it follows that $\Lambda_{t}(x \mid t)$ is of $B . V$. in $t$ on I for each $x$ in $S$, and $\operatorname{Var}_{t \in I}\left[\Lambda_{t}(x \mid t)\right]$ is bounded in $x$ on $S$.

2. Having discussed the functional $\Lambda$, we now consider the transformation $T$ of (1.1). In the present section we shall not need to assume that $\Lambda$ is of smooth variation, but we shall need to make $T$ take points of $C$ into points of $C$. Thus we assume that for each fixed $x$ in $C, \Lambda(x \mid t)$ is continuous in $t$ on $I$ and vanishes at $t=0$. This can be more simply stated thus:

$$
\Lambda(x \mid \cdot) \in C
$$

when $x \in C$.

We shall also assume that $\Lambda$ satisfies the Lipschitz condition

$$
\|\Lambda(x \mid \cdot)-\Lambda(y \mid \cdot)\|\|\leqq \lambda\| x-y \mid \|
$$

when $(x, y) \in C^{2}$ for fixed $\lambda$ satisfying

$$
0<\lambda<1
$$

With these hypotheses we shall prove in the following lemma that $T$ is 1-to-1:

LEMMA 2. Let $\Lambda(x \mid t)$ be a functional defined on $C \otimes I$ which satisfies (2.1) and (2.2) with (2.3). Then $T$ defined as in (1.1):

$$
T: y=x+\Lambda(x \mid \cdot)
$$

is a 1-to-1 transformation which takes the whole of $C$ into the whole of $C$.

To establish this, we let $x_{0}=y$, and in general let

$$
x_{n}=y-\Lambda\left(x_{n-1} \mid \cdot\right) \text {, }
$$

$$
n=1,2, \cdots \text {. }
$$

Since $\Lambda(x \mid \cdot)$ is in $C$ when $x$ is in $C$, each of the functions $x_{1}, x_{2}, \cdots$ is in $C$. Then

$$
x_{n}-x_{n-1}=y-\Lambda\left(x_{n-1} \mid \cdot\right)-y+\Lambda\left(x_{n-2} \mid \cdot\right)
$$

and by (2.2),

$$
\left\|\left|x_{n}-x_{n-1}\right|\right\|=\left\|\left|\Lambda\left(x_{n-1} \mid \cdot\right)-\Lambda\left(x_{n-2} \mid \cdot\right)\right|\right\| \leqq \lambda\left\|\mid x_{n-1}-x_{n-2}\right\| .
$$

Thus

$$
\left\|\left|x_{n}-x_{n-1}\right|\right\| \leqq \lambda^{n-1}\left\|\mid x_{1}-x_{0}\right\|
$$

and by (2.3) 


$$
\sum_{n=1}^{\infty}\left\|\left|x_{n}-x_{n-1} \|\right|\right. \text { converges. }
$$

Thus $\sum_{n=1}^{\infty}\left[x_{n}(t)-x_{n-1}(t)\right]$ converges uniformly, and $\lim _{n \rightarrow \infty} x_{n}(t)$ exists uniformly. Call it $x(t)$. Then $x \in C$ and

$$
\lim _{n \rightarrow \infty}\left\|\left|x_{n}-x \|\right|=0 .\right.
$$

Now from (2.5), we have

$$
x(t)=y(t)-\lim _{n \rightarrow \infty} \Lambda\left(x_{n-1} \mid t\right),
$$

where the latter limit is uniform. But it follows from (2.2) and (2.6) that

$$
\lim _{n \rightarrow \infty} \Lambda\left(x_{n-1} \mid t\right)=\Lambda(x \mid t)
$$

so

$$
x(t)=y(t)-\Lambda(x \mid t)
$$

and (2.4) is satisfied.

Thus to each element of $C$ there corresponds at least one element $x$ in $C$ satisfying (2.4).

Moreover, this is the only such $x$, for if

$$
x+\Lambda(x \mid \cdot)=y=x^{*}+\Lambda\left(x^{*} \mid \cdot\right),
$$

then $x-x^{*}=\Lambda\left(x^{*} \mid \cdot\right)-\Lambda(x \mid \cdot)$, and

$$
\left\|\left|x-x^{*}\|=\| \Lambda\left(x^{*} \mid \cdot\right)-\Lambda(x \mid \cdot)\left\|\left|\leqq \lambda\left\|x-x^{*}\right\|\right|\right.\right.\right.
$$

which can hold only if $\left\|x-x^{*}\right\| \mid=0$ or $x(t) \equiv x^{*}(t)$.

Thus each $x$ in $C$ defines a unique $y$ in $C$ and each $y$ in $C$ defines a unique $x$ in $C$, so $T$ is a 1-to-1 transformation taking the whole of $C$ into the whole of $C$.

3. We next develop $n$-dimensional approximations to the transformation (2.4). Let $x_{n}(t)$ belong to $C$ and be the $n$-dimensional polygonalization of $x(t)$; that is, let

$$
x_{n}\left(\frac{k}{n}\right)=x\left(\frac{k}{n}\right), \quad k=0,1,2, \cdots, n,
$$

while $x_{n}(t)$ is a linear function on each interval $(k-1) / n \leqq t \leqq k / n$. The notation $[x]_{n}$ will also be used to denote $x_{n}$. Thus

$$
x_{n}(t)=[x(t)]_{n}=\sum_{k=1}^{n} \alpha_{n, k}(t) x\left(\frac{k}{n}\right)
$$

where 


$$
\alpha_{n, k}(t)= \begin{cases}1-|n t-k| & \text { for }(k-1) / n \leqq t<(k+1) / n \\ 0 & \text { elsewhere. }\end{cases}
$$

Let $T_{n}$ be the transformation

$$
T_{n}: y(t)=[x(t)]_{n}+\left[\Lambda\left([x]_{n} \mid t\right)\right]_{n}
$$

or

$$
T_{n}: \quad y=x_{n}+\left[\Lambda\left(x_{n} \mid \cdot\right)\right]_{n} .
$$

It is clear that $y=y_{n}$ under these circumstances, and we may thus write

$$
T_{n}: \quad y_{n}=x_{n}+\left[\Lambda\left(x_{n} \mid \cdot\right)\right]_{n} .
$$

If we let

$$
a_{k} \equiv a_{k}^{(n)} \equiv x\left(\frac{k}{n}\right)=x_{n}\left(\frac{k}{n}\right)
$$

and

$$
b_{k} \equiv b_{k}^{(n)} \equiv y\left(\frac{k}{n}\right)=y_{n}\left(\frac{k}{n}\right)
$$

we have from (3.4)

$$
b_{k}^{(n)}=a_{k}^{(n)}+f_{k}^{(n)}\left(a_{1}^{(n)}, \ldots, a_{n}^{(n)}\right),
$$

where

$$
f_{k}^{(n)}\left(a_{1}, \cdots, a_{n}\right)=\Lambda\left(x_{n} \mid \frac{k}{n}\right)
$$

or (in the notation of (3.3)),

$$
f_{k}^{(n)}\left(a_{1}, \cdots, a_{n}\right)=\Lambda\left(\sum_{j=1}^{n} a_{j} \alpha_{n, j}(\cdot) \mid \frac{k}{n}\right) .
$$

(The superscript $n$ will cause no confusion since there are no $n$th derivatives in this paper.)

Let $a$ or $a^{(n)}$ denote the vector $\left[a_{1}^{(n)}, a_{2}^{(n)}, \cdots, a_{n}^{(n)}\right]$ and $b$ or $b^{n}$ the vector $\left[b_{1}, \cdots, b_{n}\right]$ and $f^{(n)}(a)$ the vector $\left[f_{1}^{(n)}\left(a_{1}, \cdots, a_{n}\right), \cdots, f_{n}^{(n)}\left(a_{1}, \cdots, a_{n}\right)\right]$. Let us define the $n$-dimensional norm

$$
\|a\|\left\|^{(n)} \equiv\right\| a^{(n)} \|^{(n)}=\max _{j=1, \cdots, n}\left|a_{j}^{(n)}\right| .
$$

Clearly

$$
\left\||| x_{n}\right\|\|=\| a^{(n)} \mid \|(n)
$$


and

$$
\left\|y_{n}\right\||=\|b \mid\|(n)
$$

and

$$
\left\|f^{(n)}(a)\right\|\left\|^{(n)}=\right\|\left\|\left[\Lambda\left(x_{n} \mid \cdot\right)\right]_{n}\right\| \cdot
$$

Thus

$$
\begin{aligned}
\left\|f^{(n)}(a)-f^{(n)}\left(a^{*}\right)\right\|(n) & =\left\|\left[\Lambda\left(x_{n} \mid \cdot\right)-\Lambda\left(x_{n}^{*} \mid \cdot\right)\right]_{n}\right\| \\
& \leqq\left\|\Lambda\left(x_{n} \mid \cdot\right)-\Lambda\left(x_{n}^{*} \mid \cdot\right)\right\| \| \\
& \leqq \lambda\|\| x_{n}-x_{n}^{*}\left\|\left|=\lambda\left\|\left|a-a^{*} \|\right|^{(n)}\right.\right.\right.
\end{aligned}
$$

and we have the Lipschitz condition

$$
\left\|f^{(n)}(a)-f^{(n)}\left(a^{*}\right)\right\|\left\|^{(n)} \leqq \lambda\right\| a^{(n)}-a^{*(n)} \|\left.\right|^{(n)}
$$

analogous to the Lipschitz condition for $\Lambda(x)$.

Now (3.6) takes each vector $a$ into a vector $b$. However, let $b$ be given and let us solve (3.6) for $a$. Dropping superscripts, we write (3.6) as

$$
T_{n}: \quad b=a+f(a) .
$$

Since $b$ is given, choose $A_{0}=b$ and

$$
A_{v}=b-f\left(A_{v-1}\right), \quad \quad v=1,2, \cdots \text {. }
$$

By the Lipschitz condition,

$$
\left\|A_{v}-A_{v-1}\right\|(n)=\left\|f\left(A_{v-1}\right)-f\left(A_{v-2}\right)\right\|(n) \leqq \lambda\left\|A_{v-1}-A_{v-2}\right\| \|^{(n)}
$$

and (dropping superscripts on norms),

$$
\left\|A_{v}-A_{v-1}\right\| \leqq \lambda^{v-1}\left\|A_{1}-A_{0}\right\|,
$$

and $\sum\left\|A_{v}-A_{v-1}\right\| \|$ converges. Hence

$$
\lim _{0 \rightarrow \infty} \sum_{k=0}^{\infty}\left\|A_{k}-A_{k-1}\right\| \mid=0
$$

But

$$
\left\|\left(\sum_{k=v+1}^{v+p}\left(A_{k}-A_{k-1}\right)\right)\right\| \leqq \sum_{k=v}^{\infty}\left\|A_{k}-A_{k-1}\right\|,
$$

so

$$
\lim _{r \rightarrow \infty}\left\|A_{v+p}-A_{v}\right\|=0 \text { uniformly for positive } p \text {; }
$$

and $\lim _{v \rightarrow \infty} A_{v}$ exists. Call it $a$. Since $f$ satisfies a Lipschitz condition, it is 
continuous, and taking limits as $v \rightarrow \infty$ in (3.10), we obtain

$$
a=b-f(a) .
$$

Thus this vector $a$ satisfies (3.6).

Moreover this vector $a$ is the only solution of (3.6), for if we had two solutions we would have

$$
a+f(a)=b=a^{*}+f\left(a^{*}\right)
$$

and

$$
a-a^{*}=f\left(a^{*}\right)-f(a)
$$

and

$$
\left\|a-a^{*}\right\| \mid=\left\|f(a)-f\left(a^{*}\right)\right\| \leqq \lambda\left\|a-a^{*}\right\|
$$

so that $\left\|a-a^{*}\right\|=0$ and $a=a^{*}$.

Hence the transformation $T_{n}$ is 1 -to- 1 , taking all $n$-dimensional vectors $a$ into all $n$-dimensional vectors $b$.

This can also be stated thus: $T_{n}$ is 1 -to- 1 , taking all $n$-dimensional polygonal functions $x_{n}$ of $C$ into all $n$-dimensional polygonal functions $y_{n}$ of $C$. Thus a transformation $T_{n}^{-1}$ is defined which takes all $n$-dimensional polygonal functions $y_{n}$ of $C$ into all $n$-dimensional polygonal functions $x_{n}$ of $C$ :

$$
T_{n}^{-1}\left(y_{n}\right)=x_{n}
$$

Moreover if $y_{n}^{*}$ has the transform $x_{n}^{*}$, by (3.11) we have

$$
y_{n}=x_{n}+\left[\Lambda\left(x_{n} \mid \cdot\right)\right]_{n} \text { and } y_{n}^{*}=x_{n}^{*}+\left[\Lambda\left(x_{n}^{*} \mid \cdot\right)\right]_{n},
$$

so

$$
x_{n}^{*}-x_{n}=y_{n}^{*}-y_{n}-\left[\Lambda\left(x_{n}^{*} \mid \cdot\right)\right]_{n}+\left[\Lambda\left(x_{n} \mid \cdot\right)\right]_{n},
$$

and by (3.9)

$$
\left\|x_{n}^{*}-x_{n}\right\| \leqq\left\|y_{n}^{*}-y_{n}\right\| \mid+\lambda\|\| x_{n}^{*}-x_{n} \|
$$

so that

$$
\left\|x_{n}^{*}-x_{n}\right\| \mid \leqq(1-\lambda)^{-1}\left\|y_{n}^{*}-y_{n}\right\| .
$$

Thus we have the following lemma.

Lemma 3. Let $\Lambda(x \mid t)$ be defined on $C \otimes I$ and satisfy (2.1), (2.2), (2.3); let $n$ be fixed, and let $f_{k}^{(n)}\left(a_{1}, \cdots, a_{n}\right)$ be defined as in (3.7). Then $f$ satisfies the Lipschitz condition (3.9) (with (3.8)). Moreover the transformation $T_{n}: b=a$ $+f(a)$ is a 1-to-1 transformation of the whole $n$-dimensional space into itself; 
or if we interpret $T_{n}$ as in (3.4), it takes the set of all n-dimensional polygonal functions in $C$ into itself.

Finally if $T_{n}^{-1}$ (see (3.11)) denotes the inverse of $T_{n}$ (see 3.4)), we have for all polygonal functions $y_{n}$ and $y_{n}^{*}$ in C the Lipschitz condition

$$
\left\|T_{n}^{-1}\left(y_{n}^{*}\right)-T_{n}^{-1}\left(y_{n}\right)\right\| \leqq(1-\lambda)^{-1}\left\|y_{n}^{*}-y_{n}\right\| .
$$

Here $\lambda$ is the number given in the hypothesis (2.2).

4. We now use Lemma 3 to obtain our first preliminary theorem on the transformation of Wiener integrals.

Theorem I. Let $\Lambda(x \mid t)$ be defined on $C \otimes I$ and satisfy (2.1), (2.2), (2.3) and let $f_{k}^{(n)}\left(a_{1}, \cdots, a_{n}\right)$ be defined by (3.7). Let $\partial f_{j}^{(n)} / \partial a_{k}$ exist and be continuous for all $j, k, n$ and all values of $a_{1}, a_{2}, \cdots, a_{n}$. Let

$$
\left.\Delta_{n} \equiv \Delta_{n}(x) \equiv \text { determinant of }\left\{\frac{\partial f_{j}^{(n)}}{\partial a_{k}^{(n)}}+\delta_{j, k}\right\}\right]_{\substack{(n)=x(k / n) \\ k}}(j, k=1, \cdots, n) .
$$

Let $F[y]$ be any functional for which $F\left[y_{n}\right]$ is summable over $C$. Then

$$
\begin{array}{rl}
\int_{C}^{w} F\left(y_{n}\right) d_{w} y=\int_{C}^{w} & F\left\{x_{n}+\left[\Lambda\left(x_{n} \mid \cdot\right)\right]_{n}\right\} \\
& \cdot \exp \left\{-2 n \sum_{j=1}^{n}\left[x\left(\frac{j}{n}\right)-x\left(\frac{j-1}{n}\right)\right]\left[\Lambda\left(x_{n} \mid \frac{j}{n}\right)\right.\right. \\
& \left.\left.-\Lambda\left(x_{n} \mid \frac{j-1}{n}\right)\right]\right\} \\
\cdot & -\left|\Delta_{n}(x)\right| d_{w} x .
\end{array}
$$

We prove this theorem by evaluating both sides of (4.2) in terms of $n$-dimensional Lebesgue integrals. Since $F\left(y_{n}\right)$ depends only on the values of $y(\cdot)$ at the $n$ points $1 / n, 2 / n, \cdots, n / n$, it follows that there exists a function $H\left(\eta_{1}, \cdots, \eta_{n}\right)$ of $n$ real variables such that

$$
F\left[\sum_{j=1}^{n} \alpha_{n, j}(\cdot) \eta_{j}\right]=H\left(\eta_{1}, \cdots, \eta_{n}\right) ;
$$

that is, such that

$$
F\left(y_{n}\right)=H\left[y\left(\frac{1}{n}\right), \cdots, y\left(\frac{n}{n}\right)\right] .
$$


Then by Wiener's general theory,

$$
\begin{aligned}
\int_{C}^{w} F\left(y_{n}\right) d_{w} y=\pi^{-n / 2} n^{n / 2} \int_{-\infty}^{\infty} & \cdots \int_{-\infty}^{\infty} H\left(\eta_{1}, \cdots, \eta_{n}\right) \\
& \cdot \exp \left\{-n \sum_{j=1}^{n}\left(\eta_{j}-\eta_{j-1}\right)^{2}\right\} d \eta_{1} \cdots d \eta_{n}
\end{aligned}
$$

where for convenience we use $\eta_{0}=0$.

Now subject the $\eta$ 's to the 1-to-1 transformation of $\$ 3$ (Lemma 3) $\eta_{j}=$ $\xi_{j}+f_{j}^{(n)}\left(\xi_{1}, \cdots, \xi_{n}\right)$ where

$$
f_{j}^{(n)}\left(\xi_{1}, \cdots, \xi_{n}\right)=\Lambda\left(\sum_{k=1}^{n} \xi_{k} \alpha_{n, k}(\cdot) \mid \frac{j}{n}\right) .
$$

Under this transformation the second member of (4.4) becomes

$$
\begin{aligned}
\left(\frac{n}{\pi}\right)^{n / 2} \int_{-\infty}^{\infty} \cdots \int_{-\infty}^{\infty} H\left(\xi_{1}+f_{1}^{(n)}\left(\xi_{1}, \cdots, \xi_{n}\right), \cdots, \xi_{n}+f_{n}^{(n)}\left(\xi_{1}, \cdots, \xi_{n}\right)\right) \\
(4.5) \cdot \exp \left\{-n \sum_{j=1}^{n}\left(\xi_{j}-\xi_{j-1}\right)^{2}\right\} \exp \left\{-2 n \sum_{j=1}^{n}\left(\xi_{j}-\xi_{j-1}\right)\left(f_{j}^{(n)}-f_{j-1}^{(n)}\right)\right\} \\
\cdot \exp \left\{-n \sum_{j=1}^{n}\left(f_{j}^{(n)}-f_{j-1}^{(n)}\right)^{2}\right\}\left|\operatorname{det}\left\{\frac{\partial f_{j}^{(n)}}{\partial \xi_{k}}+\delta_{j, k}\right\}_{j, k=1,2, \cdots, n}\right| d \xi_{1} \cdots d \xi_{n},
\end{aligned}
$$

where we have used $f_{0}^{(n)}=\xi_{0}=0$.

When we transform (4.5) back to a Wiener integral over $C$, we obtain the right-hand member of (4.2).

5. We shall of course seek to obtain an infinite-dimensional theorem from Theorem I by taking the limit as $n \rightarrow \infty$. Before we do this, we must study $\Lambda\left(x_{n} \mid \cdot\right)$ and its behavior as $n \rightarrow \infty$ more extensively. We now require the assumption of $\$ 1$ that $\Lambda$ is of smooth variation.

LEMMA 4. Let $\Lambda(x \mid t)$ be of smooth variation on $S \otimes I$ (where $S$ is a (uniformly) open convex subset of $C$ ) and let its kernel $K$ have a partial derivative $K_{t}(x \mid t, s)$ which is continuous for all $(x, t, s)$ in $S \otimes I^{2}$ (uniform topology for $x$ ), and let $\partial \Lambda\left(x_{0} \mid t\right) / \partial t$ exist and be continuous for all $t$ in $I$ and some fixed $x_{0}$ in $S$. Then if $x_{n}$ is the n-dimensional polygonalization of $x$ (given by (3.2) and (3.3)), for each $x$ in $S$ we have

$$
\lim _{n \rightarrow \infty} n \sum_{j=1}^{n}\left[\Lambda\left(x_{n} \mid \frac{j}{n}\right)-\Lambda\left(x_{n} \mid \frac{j-1}{n}\right)\right]^{2}=\int_{0}^{1}\left[\frac{\partial}{\partial t} \Lambda(x \mid t)\right]^{2} d l .
$$

To see this, we first note by Corollary 4 to Lemma 1 that $\Lambda_{t}(x \mid t)$ exists and is continuous throughout $S \otimes I$ and that if $x \in S$ then for sufficiently large $n, x_{n} \in S$. By the mean value theorem, the limitand of (5.1) is equal to 


$$
\frac{1}{n} \sum_{j=1}^{n}\left\{\frac{\partial}{\partial t} \Lambda\left(x_{n} \mid t_{j, n}\right)\right\}^{2}, \quad \frac{j-1}{n}<t_{j, n}<\frac{j}{n} .
$$

Now

$$
\left|\int_{0}^{1}\left\{\frac{\partial}{\partial t} \Lambda(x \mid t)\right\}^{2} d t-\frac{1}{n} \sum_{j=1}^{n}\left\{\frac{\partial}{\partial t} \Lambda\left(x_{n} \mid t_{j, n}\right)\right\}^{2}\right| \leqq\left|A_{1}\right|+\left|A_{2}\right|,
$$

where

$$
A_{1}=\int_{0}^{1}\left\{\frac{\partial}{\partial t} \Lambda(x \mid t)\right\}^{2} d t-\frac{1}{n} \sum_{j=1}^{n}\left\{\frac{\partial}{\partial t} \Lambda\left(x \mid t_{j, n}\right)\right\}^{2}
$$

and

$$
A_{2}=\frac{1}{n} \sum_{j=1}^{n}\left\{\frac{\partial}{\partial t} \Lambda\left(x \mid t_{i, n}\right)\right\}^{2}-\frac{1}{n} \sum_{j=1}^{n}\left\{\frac{\partial}{\partial t} \Lambda\left(x_{n} \mid t_{j, n}\right)\right\}^{2} .
$$

Now let $\epsilon>0$. Then by definition of the integral there exists an index $N_{\epsilon, x}^{\prime}$ such that $\left|A_{1}\right|<\epsilon / 2$ for $n>N_{\epsilon, x}^{\prime}$. Also by Corollary 4 to Lemma $1, \Lambda_{t}(x \mid \cdot)$ is continuous (uniform topology), and there exists an index $N_{e, x}^{\prime \prime}$ such that

$$
\left|\left\{\frac{\partial}{\partial t} \Lambda(x \mid t)\right\}^{2}-\left\{\frac{\partial}{\partial t} \Lambda\left(x_{n} \mid t\right)\right\}^{2}\right|<\frac{\epsilon}{2} \quad \text { when } n>N_{\epsilon, x}^{\prime \prime}, t \text { in } I .
$$

Hence $\left|A_{2}\right|<\epsilon / 2$ for $n>N_{\epsilon, x}^{\prime \prime}$ and Lemma 4 holds.

Lemma 5. Let $\Lambda$ and $S$ satisfy the hypotheses of Lemma 4 and in addition assume that $\Lambda_{t}\left(x_{0} \mid t\right)$ is of $B . V$. in $I$ and $K_{t}[x \mid t, s]$ is of $B . V$. in $t$ for $(x, s) \in S \otimes I$ and that $\max _{t \in I}\left|K_{t}[x \mid t, s]\right|$ and $\operatorname{Var}_{t \in I}\left[K_{t}(x \mid t, s)\right]$ are bounded $(x, s)$ on $S \otimes I$. Then for each $x$ in $S$,

$$
\begin{gathered}
\lim _{n \rightarrow \infty} n \sum_{j=1}^{n}\left[\Lambda\left(x_{n} \mid \frac{j}{n}\right)-\Lambda\left(x_{n} \mid \frac{j-1}{n}\right)\right]\left[x\left(\frac{j}{n}\right)-x\left(\frac{j-1}{n}\right)\right] \\
=\int_{0}^{1}\left[\frac{\partial}{\partial t} \Lambda(x \mid t)\right] d x(t) .
\end{gathered}
$$

To establish this lemma, we note from Corollary 4 of Lemma 1 that $\Lambda_{t}(x \mid t)$ exists and is continuous throughout $S \otimes I$ and is of B.V. in $t$ for each $x$ in $S$. By the mean value theorem, the limitand of (5.4) is equal to

Thus

$$
\begin{gathered}
P_{n}(x) \equiv \sum_{j=1}^{n}\left[\frac{\partial}{\partial t} \Lambda\left(x_{n} \mid t_{j, n}\right)\right]\left[x\left(\frac{j}{n}\right)-x\left(\frac{j-1}{n}\right)\right], \\
\frac{j-1}{n}<t_{j, n}<\frac{j}{n} .
\end{gathered}
$$




$$
\left|\int_{0}^{1}\left[\frac{\partial}{\partial t} \Lambda(x \mid t)\right] d x(t)-P_{n}(x)\right| \leqq\left|B_{1}\right|+\left|B_{2}\right|,
$$

where

$$
\begin{aligned}
& B_{1} \equiv B_{1}(x, n) \\
& \equiv \int_{0}^{1}\left[\frac{\partial}{\partial t} \Lambda(x \mid t)\right] d x(t)-\sum_{1}^{n}\left[\frac{\partial}{\partial t} \Lambda\left(x \mid t_{j, n}\right)\right]\left[x\left(\frac{j}{n}\right)-x\left(\frac{j-1}{n}\right)\right], \\
& B_{2} \equiv B_{2}(x, n) \\
& \quad \equiv \sum_{1}^{n}\left[\frac{\partial}{\partial t} \Lambda\left(x \mid t_{j n}\right)-\frac{\partial}{\partial t} \Lambda\left(x_{n} \mid t_{j, n}\right)\right]\left[x\left(\frac{j}{n}\right)-x\left(\frac{j-1}{n}\right)\right] .
\end{aligned}
$$

Now by the definition of a Riemann-Stieltjes integral the expression $B_{1}$ approaches zero for each $x$ in $C$ as $n \rightarrow \infty$. Moreover by Corollary 4 to Lemma 1 ,

$$
\begin{aligned}
\Omega_{j, n}(x) & \equiv \frac{\partial}{\partial t} \Lambda\left(x \mid t_{j, n}\right)-\frac{\partial}{\partial t} \Lambda\left(x_{i} \mid t_{i, n}\right) \\
& =\int_{0}^{1} d V \int_{0}^{1} K_{t}\left[x_{n}+\left(x-x_{n}\right) V \mid t_{i, n}, s\right]\left[x(s)-x_{n}(s)\right] d s .
\end{aligned}
$$

Also

$$
\begin{aligned}
B_{2} & =\sum_{j=1}^{n} \Omega_{j, n}(x)\left[x\left(\frac{j}{n}\right)-x\left(\frac{j-1}{n}\right)\right] \\
& =\Omega_{n, n}(x) x(1)+\sum_{j=1}^{n-1}\left[\Omega_{j, n}-\Omega_{j+1, n}\right] x\left(\frac{j}{n}\right),
\end{aligned}
$$

so

$$
\begin{aligned}
\left|B_{2}\right| \leqq & \left|\Omega_{n, n}(x) x(1)\right|+\sum_{j=1}^{n-1}\left|x\left(\frac{j}{n}\right)\right| \int_{0}^{1} d V \int_{0}^{1} \mid K_{t}\left[x_{n}+\left(x-x_{n}\right) V \mid t_{i, n}, s\right] \\
& -K_{t}\left[x_{n}+\left(x-x_{n}\right) V \mid t_{i+1, n}, s\right]|\cdot| x(s)-x_{n}(s) \mid d s \\
\leqq & \left|\Omega_{n, n}(x) x(1)\right|+\max _{t \in I}|x(t)| j \cdot \int_{0}^{1} d V \int_{0}^{1} \operatorname{Var}_{t \in I}\left[K_{t}\left[x_{n}+\left(x-x_{n}\right) V \mid t, s\right]\right. \\
& \cdot\left|x(s)-x_{n}(s)\right| d s \\
\leqq & \left\{\max _{(t, s, V) \in I^{3}}\left|K_{t}\left[x_{n}+\left(x-x_{n}\right) V \mid t, s\right]\right| \cdot|x(1)|+\|x\|\right. \\
& \left.\cdot \max _{s, V \in I^{2}} \operatorname{Var}_{t \in I}\left[K_{t}\left[x_{n}+\left(x-x_{n}\right) V \mid t, s\right]\right]\right\} \cdot\left\|x-x_{n}\right\| \cdot .
\end{aligned}
$$


But the last factor of the product approaches 0 as $n \rightarrow \infty$ since $x(t)$ is uniformly continuous; while the first factor remains bounded because of the hypotheses on $K_{t}$. Thus $\left|B_{2}\right| \rightarrow 0$ as $n \rightarrow \infty$, and by (5.5)

$$
P_{n}(x) \rightarrow \int_{0}^{1}\left[\frac{\partial}{\partial t} \Lambda(x \mid t)\right] d x(t),
$$

so the lemma is established.

CoRollary. The limit in (5.4) is approached boundedly in $S$ if $S$ is bounded in the uniform topology and contains with each $x$ all its polygonalizations $x_{n}$.

This follows from the inequalities

$$
\begin{aligned}
\left|P_{n}(x)\right| & =\left|\left[\frac{\partial}{\partial t} \Lambda\left(x_{n} \mid t_{n, n}\right)\right] x(1)+\sum_{j=1}^{n-1}\left[\frac{\partial}{\partial t} \Lambda\left(x_{n} \mid t_{j, n}\right)-\frac{\partial}{\partial t} \Lambda\left(x_{n} \mid t_{j+1, n}\right)\right] x\left(\frac{j}{n}\right)\right| \\
& \leqq\left|\frac{\partial}{\partial t} \Lambda\left(x_{n} \mid t_{n, n}\right)\right| \cdot|x(1)|+\||x|\left|\sum_{j=1}^{n-1}\right| \frac{\partial}{\partial t} \Lambda\left(x_{n} \mid t_{j, n}\right)-\frac{\partial}{\partial t} \Lambda\left(x_{n} \mid t_{j+1, n}\right) \mid \\
& \leqq\|x\| \cdot\left\{\left|\frac{\partial}{\partial t} \Lambda\left(x_{n} \mid t_{n, n}\right)\right|+\operatorname{Var}_{t \in I}\left[\frac{\partial}{\partial t} \Lambda\left(x_{n} \mid t\right)\right]\right\}
\end{aligned}
$$

together with Corollary 4 of Lemma 1.

6. We next study the approximation of the Fredholm determinant by determinants involving polygonalized functions.

LEMMA 6. Let $\Lambda$ and $S$ satisfy the hypotheses of Lemma 4, let $\max _{(t, s) \in I^{2}}|K(x \mid t, s)|$ be bounded in every uniformly bounded subset of $S$, and let

$$
\Delta_{n} \equiv \Delta_{n}(x) \equiv \operatorname{det}\left\{\frac{\partial f_{i}^{(n)}}{\partial a_{k}^{(n)}}+\delta_{j, k}\right\}, \quad i, k=1, \cdots, n,
$$

where

$$
a_{k}^{(n)}=x\left(\frac{k}{n}\right)=x_{n}\left(\frac{k}{n}\right),
$$

$$
f_{j}^{(n)}\left(a_{1}^{(n)}, \cdots, a_{n}^{(n)}\right)=\Lambda\left(x_{n} \mid \frac{j}{n}\right)=\Lambda\left(\sum_{k=1}^{n} a_{k}^{(n)} \alpha_{n, k}(\cdot) \mid \frac{j}{n}\right)
$$

and

$$
\alpha_{n, k}(t)= \begin{cases}1-|n t-k| & \text { for }(k-1) / n \leqq t<(k+1) / n \\ 0 & \text { elsewhere. }\end{cases}
$$

Then for each $x$ in $S$ 


$$
\lim _{n \rightarrow \infty} \Delta_{n}(x)=D(x)
$$

where $D(x)$ is the Fredholm determinant of $K(x \mid t, s)$ corresponding to the value $\lambda=-1$ :

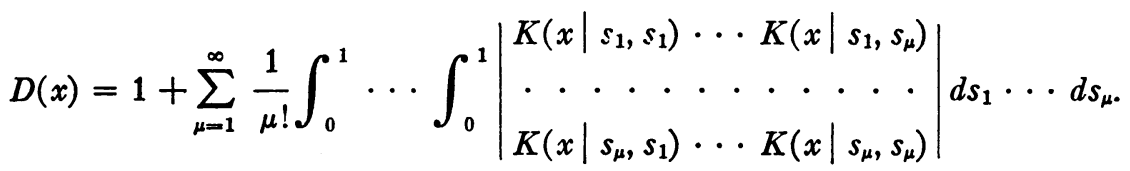

For the proof, we note from (6.1) and (1.7) that

$$
\begin{aligned}
\frac{\partial f_{j}^{(n)}}{\partial a_{k}^{(n)}} & =\int_{0}^{1} K\left(\sum_{\nu=1, v \neq k}^{n} a_{\nu}^{(n)} \alpha_{n, \nu}(\cdot)+a_{k}^{(n)} \alpha_{n, k}(\cdot) \mid \frac{j}{n}, s\right) \alpha_{n, k}(s) d s \\
& =\int_{0}^{1} K\left(x_{n} \mid \frac{j}{n}, s\right) \alpha_{n, k}(s) d s \\
& =\int_{(k-1) / n}^{(k+1) / n} K\left(x_{n} \mid \frac{j}{n}, s\right)(1-|n s-k|) d s .
\end{aligned}
$$

For convenience we write

$$
A_{j, k}^{(n)}(x)=\int_{(k-1) / n}^{(k+1) / n} K\left(x_{n} \mid \frac{j}{n}, \eta\right)(1-|n \eta-k|) d \eta
$$

so that $\Delta_{n}(x)$ may be written as

$$
\Delta_{n}(x)=\operatorname{det}\left\{A_{j, k}^{(n)}(x)+\delta_{j, k}\right\}, \quad j, k=1, \cdots, n .
$$

The proof of Lemma 6 will depend upon two other lemmas which we next state and prove. These lemmas are entirely similar in statement and proof to two lemmas proved in [1b, Lemmas 3 and 4 ].

7. Lemma 7. Let $\Lambda, S$ and $K$ be given as in Lemma 6. With each point $(t, s)$ of $I^{2}$ associate two sequences of positive integers $\left\{j_{n}(t)\right\},\left\{k_{n}(s)\right\}$ with

$$
j_{n}(t) \leqq n, \quad 1+k_{n}(s) \leqq n, \quad n=1,2, \cdots,
$$

and assume that

$$
\lim _{n \rightarrow \infty} \frac{j_{n}(t)}{n}=t, \quad \lim _{n \rightarrow \infty} \frac{k_{n}(s)}{n}=s \quad \text { uniformly in } I .
$$

Then for each $x$ in $S$

$$
\lim _{n \rightarrow \infty} n A_{j_{n}(t), k_{n}(s)}^{(n)}(x)=K(x \mid t, s) \quad \text { uniformly for }(t, s) \in I^{2}
$$

For the proof, we first observe that 


$$
n \int_{(k-1) / n}^{(k+1) / n}(1-|n \eta-k|) d \eta=1
$$

and the integrand in (7.2) is non-negative in the range considered. Hence by (6.4)

$$
\begin{aligned}
& \left|n A_{j_{n}(t), k_{n}(s)}^{(n)}(x)-K(x \mid t, s)\right| \\
& \quad \leqq \int_{\left(k_{n}(s)-1\right) / n}^{\left(k_{n}(s)+1\right) / n}\left|K\left(x_{n} \mid \frac{j_{n}(t)}{n}, \eta\right)-K(x \mid t, s)\right| n\left(1-\left|n \eta-k_{n}(s)\right|\right) d \eta \\
& \quad \leqq \sup \left|K\left(x_{n} \mid \frac{j_{n}(t)}{n}, \eta\right)-K(x \mid t, s)\right|
\end{aligned}
$$

where the supremum is taken over

$$
t \in I, \quad s \in I, \quad \frac{k_{n}(s)-1}{n} \leqq \eta \leqq \frac{k_{n}(s)+1}{n} .
$$

For each fixed $x$ in $C$ we shall show that this supremum approaches zero as $n \rightarrow \infty$. For this purpose, note that

$$
\begin{aligned}
\left|K\left(x_{n} \mid \frac{j_{n}(t)}{n}, \eta\right)-K(x \mid t, s)\right| \leqq & \left|K\left(x_{n}\left|\frac{j_{n}(t)}{n}, \eta\right|\right)-K\left(x \mid \frac{j_{n}(t)}{n}, \eta\right)\right| \\
& +\left|K\left(x \mid \frac{j_{n}(t)}{n}, \eta\right)-K(x \mid t, s)\right| .
\end{aligned}
$$

By the definition of a functional of smooth variation, we know that $K(x \mid t, s)$ is continuous in $(x, t, s)$ on $S \otimes I^{2}$. Moreover we noted in the paragraph after the definition that this implies that for each $x$ in $S, K$ is continuous in $x$ uniformly with respect to $(t, s)$ in $I^{2}$. It is of course continuous in $(t, s)$ for each fixed $x$. Hence if $\epsilon>0$ and $x$ is any fixed element of $C$, there exists an index $N$ s such that

$$
\left|K\left(x_{n} \mid \frac{j_{n}(t)}{n}, \eta\right)-K\left(x \mid \frac{j_{n}(t)}{n}, \eta\right)\right|<\frac{\epsilon}{2} \quad \text { for } n \geqq N_{\text {. }}
$$

and

$$
\left|K\left(x \mid \frac{j_{n}(t)}{n}, \eta\right)-K(x \mid t, s)\right| 1<\frac{\epsilon}{2} \quad \text { for } n \geqq N_{\epsilon},
$$

the two inequalities holding throughout (7.3). This concludes the proof of Lemma 7.

Lemma 8. If $N$ is a fixed positive integer and $q_{1}, \cdots, q_{N}$ a permutation of $1, \cdots, N$, then with the hypotheses and notation of Lemma 7 we have for each 
$x$ in $S$

$$
\lim _{n \rightarrow \infty} \sum_{p_{1}, \cdots, p_{N}=1}^{n} \prod_{\mu=1}^{N} A_{t_{\mu}, p_{\mu}}^{(n)}(x)=\int_{0}^{1} \cdots \int_{0}^{1} \prod_{\mu=1}^{N} K\left(x \mid s_{q_{\mu}}, s_{\mu}\right) d s_{\mu} \quad\left(t_{\mu}=p_{q_{\mu}}\right) .
$$

The proof of this lemma is identical with the proof of Lemma 4 of [1b] and is therefore omitted.

Corollary to Lemma 8. For each $x$ in $S$,

$$
\begin{aligned}
& \lim _{n \rightarrow \infty} \sum_{p_{1}, \cdots, p_{N^{-1}}}^{n}\left[\operatorname{det}\left(A_{p_{i}, p_{j}}^{(n)}(x)\right)_{i, j=1, \cdots, N]}\right. \\
& =\int_{0}^{1} \cdots \int_{0}^{1}\left|\begin{array}{c}
K\left(x \mid s_{1}, s_{2}\right) \cdots \cdot K\left(x \mid s_{1}, s_{N}\right) \\
\cdot \cdot \cdot \cdot \cdot \cdot \cdot \cdot \cdot \cdot \\
K\left(x \mid s_{N}, s_{1}\right) \cdots \cdot K\left(x \mid s_{N}, s_{N}\right)
\end{array}\right| d s_{1} \cdots d s_{N} .
\end{aligned}
$$

8. We now continue with the proof of Lemma 6. By (6.4) and (7.2) we see that

$$
\left|n A_{j k}^{(n)}(x)\right| \leqq \max _{(k-1) / n \leqq \eta \leqq(k+1) / n}\left|K\left(x_{n} \mid \frac{j}{n}, \eta\right)\right|
$$

and since $K(x \mid t, s)$ is continuous in $x$ uniformly in $s$ and $t$, and for each fixed $x$ in $C$, continuous in $s$ and $t$, it follows that

$$
n A_{j, k}^{(n)}(x)
$$

for each fixed $x$ is bounded in $n, j$ and $k$. Call its bound $M(x)$. Then the remainder of the proof of Lemma 6 follows as in $[1 b, \$ 7, \mathrm{pp} .195,196]$.

Corollary to Lemma 6. The limit in (6.2) is approached boundedly in $S$ provided that $K(x \mid t, s)$ is bounded in $S \otimes I^{2}$ and $S$ contains with each $x$ all its polygonalizations $x_{n}$.

For let

$$
|K(x \mid t, s)|<B \quad \text { whenever }(x, s, t) \in S \otimes I^{2} .
$$

Then when $x \in S$, it follows from (8.1) that

$$
\left|n A_{j, k}^{(n)}(x)\right| \leqq B .
$$

But by Hadamard's lemma,

$$
\left|\operatorname{det}\left\{A_{j, k}+\delta_{j, k}\right\}\right| \leqq \prod_{j=1}^{n}\left(\sum_{k=1}^{n}\left[A_{j k}+\delta_{j, k}\right]^{2}\right)^{1 / 2},
$$

so by (6.5) and (8.3) we see that when $x \in S$, 


$$
\begin{aligned}
\left|\Delta_{n}(x)\right| & \leqq \prod_{j=1}^{n}\left(\sum_{k=1}^{n}\left(\frac{B}{n}+\delta_{j, k}\right)^{2}\right)^{1 / 2} \\
& =\prod_{j=1}^{n}\left(n \frac{B^{2}}{n^{2}}+2 \frac{B}{n}+1\right)^{1 / 2}=\prod_{j=1}^{n}\left(1+\frac{\left(2 B+B^{2}\right)}{n}\right)^{1 / 2} \\
& =\left[\left(1+\frac{\left(2 B+B^{2}\right)}{n}\right)^{n}\right]^{1 / 2} \leqq e^{\left(2 B+B^{2}\right) / 2},
\end{aligned}
$$

so $\Delta_{n}(x)$ is bounded in $S$.

9. We can now establish a limiting form of Theorem I as $n \rightarrow \infty$.

TheOREM II. Let $\Lambda(x \mid t)$ be defined on $C \otimes I$ and be of smooth variation with kernel $K(x \mid t, s)$ defined on $C \otimes I^{2}$. For some fixed $x_{0} \in C$, let $\Lambda\left(x_{0} \mid \cdot\right) \in C$ and let $\Lambda_{t}\left(x_{0} \mid t\right)$ exist and be continuous and of bounded variation in $t$ on $I$. Let $K(x \mid 0, s)=0$ on $C \otimes I$, let $\left[\int_{0}^{1}[K(x \mid t, s)]^{2} d s\right]^{1 / 2} \leqq \lambda<1$ for $(x, t)$ on $C \otimes I$, and let $K_{t}(x \mid t, s)$ exist and be continuous in $(x, t, s)$ (with respect to the uniform topology so far as $x$ is concerned) in $C \otimes I^{2}$. Let $K_{t}(x \mid t, s)$ be of $B . V$. in $t$ for $(x, s)$ in $C \otimes I$ and let $\sup _{s \in I} \operatorname{Var}_{t \in I}\left[K_{t}(x \mid t, s)\right]$ and $\max _{(s, t) \in I^{2}}\left|K_{t}(x \mid t, s)\right|$ be bounded in $x$ for every uniformly bounded subset of $C$. Let $D(x)$ be the Fredholm determinant of $K(x \mid t, s)$ corresponding to the value - 1 of the parameter: that is, let

$$
\begin{aligned}
& \text { (9.1) }
\end{aligned}
$$

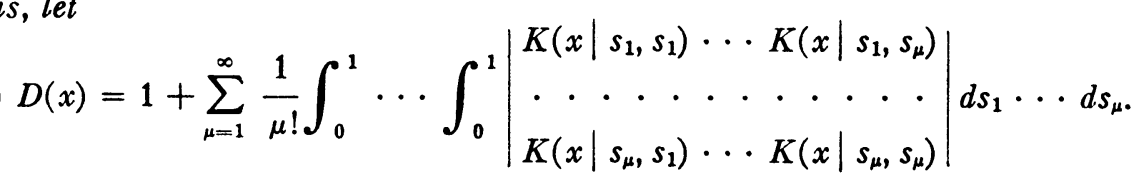

Finally, let $F(y)$ be a Wiener measurable functional over $C$. Then the following equation is true in the sense that the existence of either side implies that of the other and their equality:

$$
\begin{aligned}
\int_{C}^{w} F(y) d_{W} y & =\int_{C}^{W} F\{x+\Lambda(x \mid \cdot)\} \\
\cdot & \exp \left\{-2 \int_{0}^{1}\left[\frac{\partial}{\partial t} \Lambda(x \mid t)\right] d x(t)-\int_{0}^{1}\left[\frac{\partial}{\partial t} \Lambda(x \mid t)\right]^{2} d t\right\} \\
\cdot \mid & |D(x)| d_{W} x .
\end{aligned}
$$

We first prove this theorem under stronger hypotheses on $F$. We assume (as Case I) that $F(y)$ is continuous in the uniform topology and bounded over $C$ and that it vanishes outside an open uniform sphere $J$ whose center we take for convenience as $\Lambda(0 \mid \cdot)$; that is, the value of $\Lambda$ when $x \equiv 0$; and whose radius we shall call $R$. Thus we assume $F(y)=0$ when $y \notin J$, where $J:\|y-\Lambda(0 \mid \cdot)\| \mid<R$. We readily verify that $\Lambda$ and $F$ satisfy the hypotheses of Theorem I. For it follows from Corollary 2 of Lemma 1 that $\Lambda$ satisfies (2.1), and from Corollary 3 that $\Lambda$ satisfies (2.2) and (2.3). Hence from 
Theorem I we obtain (4.2), and we proceed to take the limit on both sides as $n \rightarrow \infty$.

The continuity of $F$ implies that $F\left(y_{n}\right) \rightarrow F(y)$ as $n \rightarrow \infty$, and its boundedness permits us to use the principle of bounded convergence and proceed to the limit. We see that the limit of the left member of (4.2) is the left member of (9.2).

We next seek to establish the same result for the right member. We note by (2.2) that $\Lambda\left(x_{n} \mid t\right) \rightarrow \Lambda(x \mid t)$ uniformly in $t$, and since $\Lambda(x \mid t)$ is uniformly continuous in $t,\left[\Lambda\left(x_{n} \mid t\right)\right]_{n} \rightarrow \Lambda(x \mid t)$ uniformly in $t$ for each $x$. Thus for fixed $x$, $F\left\{x_{n}+\left[\Lambda\left(x_{n} \mid \cdot\right)\right]_{n}\right\} \rightarrow F[x+\Lambda(x \mid \cdot)\}$, so that the left factor of the right integrand of (4.2) approaches the desired limit. We note also from Lemmas 4 and 5 that for each fixed $x$, the exponential factors in the right-hand integral approach the right limits. (In applying Lemmas 4 and 5 we may choose $S$ as any sphere in the uniform topology which has its center at the origin and contains the particular $x$ in question.) Finally, Lemma 6 (with the same choice of $S$ ) shows that the last factor of the right-hand integrand of (4.2) approaches the corresponding factor in (9.2). Thus we have convergence to the right limit for each $x$ of the integrand on the right, and we need only show that the integrand on the right of (4.2) is bounded in $x$ and $n$.

We first establish boundedness of the integrand when $x \in S_{1}$, where $S_{1}$ is an open sphere in the uniform topology about the origin with radius $R /(1-\lambda)$ :

$$
S_{1}: \quad\|x\|<\frac{R}{1-\lambda} .
$$

In $S_{1}$, by the corollary to Lemma 6 (in $\$ 8$ ), we have that $D(x)$ is bounded. The exponential factors are also bounded, by the corollary to Lemma 5 and the fact that the exponents which are sums of squares are preceded by minus signs. Moreover $F$ is bounded everywhere, so the integrand of the right member of (4.2) is bounded, when $x \in S_{1}$. Let $B$ be its bound.

Next let $x^{*}$ and $n^{*}$ be such that $x_{n^{*}}^{*}$ is in $S_{1}$ but $x^{*}$ is not in $S_{1}$. Then the integrand does not exceed $B$ when $x=x_{n^{*}}^{*}$ since the latter is in $S_{1}$. But when $n=n^{*}$, the value of the integrand when $x=x^{*}$ is the same as when $x=x_{n^{*}}^{*}$, since the right member of (4.2) depends only on $x_{n}$ and not directly on $x$. Thus the bound $B$ persists in this case, and we need to consider only the case when $x$ and $n$ are such that $x_{n} \in S_{1}$. But when $x_{n} \notin S_{1}, y_{n} \equiv x_{n}+\left[\Lambda\left(x_{n} \mid \cdot\right)\right]_{n} \notin J$, since if $y_{n}$ were in $J$, we would have $\left\|y_{n}-\Lambda(0 \mid \cdot)\right\|<R$ and by Lemma 3 (or 3.12)), we would have $\left\|x_{n}-0\right\|<R /(1-\lambda)$ and $x_{n}$ would be in $S_{1}$. Thus when $x_{n} \notin S_{1}, y_{n} \notin J$, and $F\left(y_{n}\right)=F\left[x_{n}+\left[\Lambda\left(x_{n} \mid \cdot\right)\right]_{n}\right]=0$, and the integrand on the right of (4.2) vanishes. We have therefore shown that this integrand is bounded in $x$ and $n$ for all $x$ in $C$ and all positive integers $n$ with bound $B$, and hence by bounded convergence we see that the right member of (4.2) approaches the right member of (9.2), and (9.2) is established. Thus Theorem 
II is established under the extra assumptions of Case I.

We remove the extra assumptions of Case $I$ in the usual way. (See [1a, $\S 5]$ and $[1 \mathrm{~b}, \S 11]$.)

A consideration of the steps of the proof just completed yields the following corollary to Theorem 2 .

CoRollary. If $\Gamma$ is a Wiener measurable subset of $C$, so are $T \Gamma$ and $T^{-1} \Gamma$, where $T$ is given by (1.1).

10. So far our transformation theorems have been applied only to transformations of the whole of $C$ into the whole of $C$. In order to deal with other domains and ranges, we shall next seek to establish a "local" theorem having to do with transformations of "sufficiently small" regions. Moreover in our "local" theorem the condition that $\left(\int_{0}^{1}[K(x \mid t, s)]^{2} d s\right)^{1 / 2} \leqq \lambda<1$ will be replaced by the condition that $\left(\int_{0}^{1}[K(x \mid t, s)]^{2} d s\right)^{1 / 2}$ be locally bounded and $\Lambda\left(x_{0} \mid t\right)=0$ for $t$ in $I$ and $K\left(x_{0} \mid t, s\right)=0$ for $(t, s)$ in $I^{2}$. This change to "local" hypotheses is made possible by a modification of the kernel $K$ which is described in the following lemma.

LEMma 9. Let $S$ be an open sphere in the Hilbert topology with center $x_{0}$ and radius $r$, and let $\Lambda(x \mid t)$ be of smooth variation in $S \otimes I$ with kernel $K(x \mid t, s)$. Let $\Lambda\left(x_{0} \mid t\right)=0$ for $t$ in $I$ and $K\left(x_{0} \mid t, s\right)=0$ for $(t, s)$ in $I^{2}$ and $K(x \mid 0, s)=0$ for $(x, s)$ in $S \otimes I$. Let $K_{t}(x \mid t, s)$ exist and be continuous in $(x, t, s)$ (uniform topology for $x)$ in $S \otimes I^{2}$, let $K_{t}(x \mid t, s)$ be of $B . V$. in $t$ for $(x, s)$ in $S \otimes I$, let $\max _{(s, t) \in I^{2}}|K(x \mid t, s)|, \max _{(s, t) \in I^{2}}\left|K_{t}(x \mid t, s)\right|$ and $\sup _{s \in I} \operatorname{Var}_{t \in I}\left[K_{t}(x \mid t, s)\right]$ be bounded in every uniformly bounded subset of $S$, and let $\left(\int_{0}^{1}[K(x \mid t, s)]^{2} d s\right)^{1 / 2}$ be bounded and continuous in the Hilbert topology in $S \otimes I$. Let $\psi(u)$ be a monotonic function of the non-negative real variable $u$ which has a continuous first derivative for all $u \geqq 0$ and satisfies

$$
\psi(u)= \begin{cases}1, & 0 \leqq u \leqq 1 \\ 0, & 2 \leqq u<\infty\end{cases}
$$

and let

$$
\Lambda_{\theta}^{*}(x \mid t)=\Lambda(x \mid t) \psi\left(\theta \cdot\left\|x-x_{0}\right\|\right)
$$

where

$$
\theta>2 / r
$$

and $\Lambda(x \mid t)$ is defined to be zero when $x \in S$.

Then $\Lambda_{\theta}^{*}(x \mid t)$ satisfies all the hypotheses given above for $\Lambda(x \mid t)$ with $S$ replaced by $C$, and in addition there exists a number $\theta_{0}>2 / r$ such that when $\theta>\theta_{0}, \Lambda_{\theta}^{*}(x \mid t)$ satisfies the condition

$$
\left(\int_{0}^{1}\left[K_{\theta}^{*}(x \mid t, s)\right]^{2} d s\right)^{1 / 2} \leqq \lambda<1, \quad(x, t) \in C \otimes I,
$$


where $K_{\theta}^{*}$ is the kernel of the variation of $\Lambda_{\theta}^{*}$.

To verify that $\Lambda_{\theta}^{*}$ satisfies all the hypotheses in $C \otimes I$, we first calculate the variation of $\Lambda_{\theta}^{*}$ and its kernel $K_{\theta}^{*}$. Thus if we let

$$
\phi(u)= \begin{cases}\psi^{\prime}(u) / u & \text { when } u>0, \\ 0 & \text { when } u=0\end{cases}
$$

we note that $\phi(u)$ is continuous and we have when $x \in S$, by (1.2), (1.3), and (1.4),

$$
\begin{aligned}
& \delta \Lambda_{\theta}^{*}(x|t| y) \\
& \left.=\frac{\partial}{\partial V}\left\{\Lambda(x+V y \mid t) \psi\left(\theta\left(\int_{0}^{1}\left[x(s)+V y(s)-x_{0}(s)\right]^{2} d s\right)^{1 / 2}\right)\right\}\right]_{V=0} \\
& =\delta \Lambda(x|t| y) \cdot \psi\left(\theta\left\|x-x_{0}\right\|\right) \\
& \quad+\Lambda(x \mid t) \phi\left(\theta\left\|x-x_{0}\right\|\right) \theta^{2} \int_{0}^{1}\left[x(s)-x_{0}(s)\right] y(s) d s \\
& =\psi\left(\theta\left\|x-x_{0}\right\|\right) \int_{0}^{1} K(x \mid t, s) y(s) d s \\
& \quad+\theta^{2} \Lambda(x \mid t) \phi\left(\theta\left\|x-x_{0}\right\|\right) \int_{0}^{1}\left[x(s)-x_{0}(s)\right] y(s) d s \\
& =\int_{0}^{1} K_{\theta}^{*}(x \mid t, s) y(s) d s,
\end{aligned}
$$

where

$$
\begin{aligned}
K_{\theta}^{*}(x \mid t, s)= & \psi\left(\theta\left\|x-x_{0}\right\|\right) K(x \mid t, s) \\
& +\theta^{2} \Lambda(x \mid t) \phi\left(\theta\left\|x-x_{0}\right\|\right)\left[x(s)-x_{0}(s)\right]
\end{aligned}
$$

when $(x, t, s) \in S \otimes I^{2}$.

(The second term vanishes as it should when $x=x_{0}$, for $\Lambda_{\theta}^{*} \equiv \Lambda$ in the neighborhood of this point by (10.1).) Again, if $x \in S,\left\|x-x_{0}\right\| \geqq r$ and $\theta\left\|x-x_{0}\right\|>2$ by (10.3), so $\psi\left(\theta\left\|x-x_{0}\right\|\right)=0$ by (10.1) and $\Lambda_{\theta}^{*}(x \mid t)=0$ by (10.2). Thus if we take $K_{\theta}^{*}(x \mid t, s)=0$ when $x \notin S$, we have

$$
\delta \Lambda_{\theta}^{*}(x|t| y)=\int_{0}^{1} K_{\theta}^{*}(x \mid t, s) y(s) d s \quad \text { when }(x, y, t) \in C^{2} \otimes I .
$$

Moreover (10.5) will still hold if $K$ is defined in any arbitrary way when $x \notin S$ since $\psi=\phi=0$ there. In particular, we may take $K=0$ when $x \notin S$, and we then have (10.5) and (10.6) holding universally.

Now continuity of $K_{\theta}^{*}(x \mid t, s)$ in $(x, t, s)$ (in the uniform topology for $x$ ) follows from (10.5) at all points where $K(x \mid t, s)$ is continuous; that is, unless 
$\left\|x-x_{0}\right\|=r$. But when $\left\|x-x_{0}\right\|=r, \theta\left\|x-x_{0}\right\|>2$, and this is also true in some uniform neighborhood of the point $x$, so that $\psi=\phi=0$ and $K_{\theta}^{*}(x \mid t, s)=0$ throughout some neighborhood of the point. Then even on the boundary of $S$ where $\left\|x-x_{0}\right\|=r, K_{\theta}^{*}$ is continuous in $(x, t, s)$, and it follows that $\Lambda_{\theta}^{*}$ is a functional of smooth variation in $C \otimes I$ with $K_{\theta}^{*}$ as its variation kernel. We can then easily verify from (10.5) and from corollaries of Lemma 1 that $\Lambda_{\theta}^{*}$ satisfies in $C \otimes I$ all the hypotheses required of $\Lambda$ in $S \otimes I$ (keeping in mind that $K_{\theta}^{*}$ vanishes when $x$ is in a sufficiently small uniform neighborhood of a boundary point of $S$ ).

It remains to verify (10.4) for sufficiently great $\theta$. Let us therefore choose $\lambda$ on the interval $0<\lambda<1$, and hold it fixed throughout the remainder of this discussion. From (10.5) and the Minkowski inequality we have

$$
\begin{aligned}
\left(\int_{0}^{1}\left[K_{\theta}^{*}(x \mid t, s)\right]^{2} d s\right)^{1 / 2} \leqq & \psi\left(\theta\left\|x-x_{0}\right\|\right)\left(\int_{0}^{1}[K(x \mid t, s)]^{2} d s\right)^{1 / 2} \\
& +\theta^{2} \Lambda(x \mid t) \phi\left(\theta\left\|x-x_{0}\right\|\right)\left\|x-x_{0}\right\| \\
= & \psi\left(\theta\left\|x-x_{0}\right\|\right)\left(\int_{0}^{1}[K(x \mid t, s)]^{2} d s\right)^{1 / 2} \\
& +\theta \Lambda(x \mid t) \psi^{\prime}\left(\theta\left\|x-x_{0}\right\|\right) .
\end{aligned}
$$

Now because of the continuity of $\left(\int_{0}^{1}[K(x \mid t, s)]^{2} d s\right)^{1 / 2}$ (Hilbert topology in $x)$, the quantity

$$
Q(x)=\max _{t \in I}\left(\int_{0}^{1}[K(x \mid t, s)]^{2} d s\right)^{1 / 2}
$$

is continuous in $x$ (Hilbert topology), in particular at $x=x_{0}$. Since $Q\left(x_{0}\right)=0$, we can choose $\rho(\delta)>0$ (for $\delta>0$ ) such that $\rho(\delta) \rightarrow 0$ steadily when $\delta \rightarrow 0^{+}$, and $|Q(x)| \leqq \rho(\delta)$ when $\left\|x-x_{0}\right\| \leqq \delta$; so

$$
|Q(x)| \leqq \rho\left(\left\|x-x_{0}\right\|\right) \text {. }
$$

From (1.4) and the hypothesis that $\Lambda\left(x_{0} \mid t\right) \equiv 0$ and the Schwarz inequality we have

$$
\begin{aligned}
|\Lambda(x \mid t)| & \leqq \max _{V \in I}\left|Q\left(x_{0}+V\left(x-x_{0}\right)\right)\right| \cdot\left\|x-x_{0}\right\| \\
& \leqq \max _{V \in I} \rho\left(V\left\|x-x_{0}\right\|\right) \cdot\left\|x-x_{0}\right\|=\rho\left(\left\|x-x_{0}\right\|\right) \cdot\left\|x-x_{0}\right\| .
\end{aligned}
$$

Thus we have from (10.7)

$$
\begin{aligned}
& \left(\int_{0}^{1}\left[K_{\theta}^{*}(x \mid t, s)\right]^{2} d s\right)^{1 / 2} \\
& \quad \leqq\left[\psi\left(\theta\left\|x-x_{0}\right\|\right)+\theta\left\|x-x_{0}\right\| \psi^{\prime}\left(\theta\left\|x-x_{0}\right\|\right)\right] \rho\left(\left\|x-x_{0}\right\|\right)
\end{aligned}
$$


Here the first factor on the right is zero when $\theta\left\|x-x_{0}\right\| \geqq 2$, and hence in finding an estimate for the left member, we may assume that $\theta\left\|x-x_{0}\right\|<2$, so that

$$
\left(\int_{0}^{1}\left[K_{\theta}^{*}(x \mid t, s)\right]^{2}\right)^{1 / 2} \leqq\left[1+2 \max _{u \geqq 0} \psi^{\prime}(u)\right] \rho\left(\frac{2}{\theta}\right) \quad \text { for all } x \text {. }
$$

But since $\rho(u) \rightarrow 0$ as $u \rightarrow 0^{+}$, we can find $\theta_{0}$ so great that when $\theta>\theta_{0}$, the right member of (10.9) is less than $\lambda$. Thus (10.4) holds and the lemma is proved.

11. We now employ Lemma 9 to prove the following local transformation theorem.

TheOREM III. Let $S$ be an open sphere in the Hilbert topology with center $x_{0}$ and radius $r$, and let $\Lambda(x \mid t)$ be of smooth variation in $S \otimes I$ with kernel $K(x \mid t, s)$. We also make the following assumptions:

$$
\begin{aligned}
\Lambda\left(x_{0} \mid t\right) & =0 \\
K\left(x_{0} \mid t, s\right) & =0 \\
K(x \mid 0, s) & =0
\end{aligned}
$$

(11.4) If $\epsilon>0$, there exists a positive number $\delta(\epsilon)$ such that

$$
\int_{0}^{1}\left\{K\left(x_{1} \mid t_{1}, s\right)-K\left(x_{2} \mid t_{2}, s\right)\right\}^{2} d s<\epsilon
$$

whenever $\int_{0}^{1}\left[x_{1}(t)-x_{2}(t)\right]^{2} d t<\delta(\epsilon)$ and $\left|t_{1}-t_{2}\right|<\delta(\epsilon)$. $S \otimes I^{2}$

(11.5) $K_{t}(x \mid t, s)$ exists and is continuous (uniform topology for $x$ ) in

(11.6) $\int_{0}^{1}[K(x \mid t, s)]^{2} d s$ is bounded in $S \otimes I$.

(11.7) $\max _{(s, t) \in I^{2}}|K(x \mid t, s)|, \max _{(s, t) \in I^{2}}\left|K_{t}(x \mid t, s)\right|$ and in every uniformly bounded subset of $S, \max _{s \in I} \operatorname{Var}_{t \in I}\left[K_{t}(x \mid t, s)\right]$ are bounded.

Under these conditions there exists a positive number $r_{0}$ such that if $\Gamma$ is any Wiener measurable subset of the Hilbert sphere

$$
S_{0}:\left\|x-x_{0}\right\|<r_{0}
$$

then $T \Gamma$ is also Wiener measurable and the following equation holds whenever

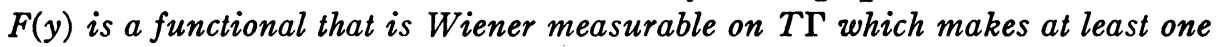
side of the equation exist:

$$
\begin{aligned}
& \int_{T \Gamma}^{w} F(y) d_{W} y=\int_{\Gamma}^{W} F\{x+\Lambda(x \mid \cdot)\} \\
& \cdot \exp \left\{-2 \int_{0}^{1}\left[\frac{\partial}{\partial t} \Lambda(x \mid t)\right] d x(t)-\int_{0}^{1}\left[\frac{\partial}{\partial t} \dot{\Lambda}(x \mid t)\right]^{2} d t\right\} \\
& \cdot|D(x)| d_{W} x
\end{aligned}
$$


Here $T \Gamma$ is understood to be the set of values assumed by $T(x)=x+\Lambda(x \mid \cdot)$ as $x$ assumes all values in $\Gamma$, and $D(x)$ is the Fredholm determinant of $K(x \mid t, s)$ as given in (9.1).

REMARK. We note that conditions (11.4) and (11.6) imply in particular that the integral $\int_{0}^{1}[K(x \mid t, s)]^{2} d s$ is continuous in $(x, t)$ (Hilbert topology for $x)$ in $S \otimes I$.

This theorem follows readily from Theorem II and Lemma 9. We first choose $\lambda$ on $0<\lambda<1$ and the function $\psi(u)$ of Lemma 9 , and then we define $\Lambda_{\theta}^{*}(x \mid t)$ by $(10.2)$ and choose $\theta_{0}$ so that when $\theta>\theta_{0}$, all the conclusions of Lemma 9 hold, including (10.4). Then $\Lambda_{\theta}^{*}(x \mid t)$ and the kernel of its variation $K_{\theta}^{*}(x \mid t, s)$ satisfy all the hypotheses imposed on $\Lambda$ and $K$ in Theorem II, so for suitable functions $F$ the conclusion (9.2) holds with $\Lambda_{\theta}^{*}$ replacing $\Lambda$. Now choose $\theta_{1}>\theta_{0}$, and choose $r_{0}=1 / \theta_{1}$. This $r_{0}$ satisfies the conditions of the theorem, for when $x \in S_{0}$, where $S_{0}:\left\|x-x_{0}\right\|<r_{0}$, we have $\theta_{1}\left\|x-x_{0}\right\|<1$ and by (10.2) and (10.1), $\Lambda_{\theta_{1}}^{*}(x \mid t)=\Lambda(x \mid t)$. Taking $\Gamma$ as any Wiener measurable subset of $S_{0}$ we have $T \Gamma$ measurable; and if $F$ is any Wiener measurable functional which vanishes outside $T \Gamma$, we see that (9.2) holds in terms of $\Lambda_{\theta}^{*}$ if either side exists, and (9.2) reduces to (11.8). Thus the theorem is established.

12. For later use we now state a theorem on linear transformations which was recently proved [1b]. (The theorem actually obtained in the paper just referred to is a slightly more general one than the one we are stating here. The present form is simpler to state and sufficient for our present purposes.)

THEOREM A. Let $x_{1}(t)$ be a function of $C$ whose first derivative $x_{1}^{\prime}(t)$ exists and is of bounded variation on $I$. Let $K_{1}(t, s)$ be defined in $I^{2}$ and assume that the following conditions are satisfied:

(12.1) $K_{1}(t, s)$ is continuous in $I^{2}$,

(12.2) For each $s$ in $I, \partial K_{1}(t, s) / \partial t$ exists and is of bounded variation for $t$ on $I$,

$$
\int_{0}^{1} \sup _{t \in I}\left|\frac{\partial}{\partial t} K_{1}(t, s)\right| d s<\infty, \quad \int_{0}^{1} \operatorname{Var}_{t \in I}\left[\frac{\partial}{\partial t} K_{1}(t, s)\right] d s<\infty .
$$

(12.4) $\mathcal{D}_{1} \neq 0$, where $\mathcal{D}_{1}$ is the Fredholm determinant of $K^{1}(t, s)$ with parameter $(-1)$.

Let $T_{1}$ be the linear transformation:

$$
T_{1}: y(t)=x(t)+x_{1}(t)+\int_{0}^{1} K_{1}(t, s) x(s) d s .
$$

Then the following equality holds whenever $U$ is any Wiener measurable subset of $C$ and $F(y)$ is a functional which is Wiener measurable on $T_{1} U$ which makes at least one side of the equation exist: 


$$
\begin{aligned}
& \int_{T_{1} V}^{W} F(y) d_{W} y=\left|\mathcal{D}_{1}\right| \int_{V}^{w} F\left[x+x_{1}+\int_{0}^{1} K_{1}(\cdot, s) x(s) d s\right] \\
& \cdot \exp \left\{-2 \int_{0}^{1}\left[x_{1}{ }^{\prime}(t)+\frac{d}{d t} \int_{0}^{1} K_{1}(t, s) x(s) d s\right] d x(t)\right. \\
&\left.-\int_{0}^{1}\left[x_{1}{ }^{\prime}(t)+\frac{d}{d t} \int_{0}^{1} K_{1}(t, s) x(s) d s\right]^{2} d t\right\} d_{W} x
\end{aligned}
$$

13. Our next goal is to prove the following theorem which is just like Theorem III except that assumptions (11.1) and (11.2) are replaced by

(13.1) $\Lambda\left(x_{0} \mid t\right) \in C, \partial \Lambda\left(x_{0} \mid t\right) / \partial t$ exists and is of bounded variation on $I$, and

$$
D\left(x_{0}\right) \neq 0 \text {. }
$$

THEOREM IV. The conclusion of Theorem III holds if the assumptions (11.1) and (11.2) are replaced by (13.1) and (13.2) and the remaining assumptions are unchanged.

The following brief outline of the proof will be helpful before we give the actual proof. Let $T_{1}$ be the linear transformation "tangent" to $T$ at $x_{0}$ :

$$
T_{1}: \quad y(t)=x(t)+\Lambda_{1}(x \mid t)
$$

where

$$
\Lambda_{1}(x \mid t)=\Lambda\left(x_{0} \mid t\right)+\int_{0}^{1} K\left(x_{0} \mid t, s\right)\left[x(s)-x_{0}(s)\right] d s
$$

and let $T_{1}^{-1}$ be its inverse. Define

$$
T_{2}=T_{1}^{-1} T \text {. }
$$

We shall show that the linear transformation (13.3) satisfies the hypotheses of Theorem A and that the transformation $T_{2}$ satisfies the hypotheses of Theorem III. On applying successively the two transformations $T_{1}$ and $T_{2}$ we shall then show that we are led to the desired result (Theorem IV) for the product $T=T_{1} T_{2}$.

We now carry out the steps of the proof.

LEMMA 10. Under the hypotheses of Theorem IV the linear transformation $T_{1}$ defined in (13.3) satisfies the hypotheses of Theorem A.

Proof. We first note by (13.1), (11.5), and (11.7) that the function

$$
x_{1}(t)=\Lambda\left(x_{0} \mid t\right)-\int_{0}^{1} K\left(x_{0} \mid t, s\right) x_{0}(s) d s
$$

is a function of $C$ whose first derivative exists and is of bounded variation on $I$. Also since $\Lambda(x \mid t)$ is of smooth variation in $S \otimes I$ with kernel $K(x \mid t, s)$ 
it follows that the kernel

$$
K_{1}(t, s)=K\left(x_{0} \mid t, s\right)
$$

satisfies condition (12.1) of Theorem A. Conditions (12.2) and (12.3) are implied by (11.5) and (11.7). Finally we note that $D_{1}=D\left(x_{0}\right) \neq 0$ and the proof of Lemma 10 is complete.

Since $D\left(x_{0}\right) \neq 0$ the linear transformation $T_{1}$ of (13.3) has a unique inverse $T_{1}^{-1}$ and by known results (see, for example [4, pp. 216-218]), the inverse is given by

(13.8) $T_{1}^{-1}: y(t)=z(t)-\Lambda\left(x_{0} \mid t\right)+\int_{0}^{1} K^{-1}(t, s)\left[z(s)-x_{0}(s)-\Lambda\left(x_{0} \mid s\right)\right] d s$,

where $K^{-1}(t, s)$ is the Volterra reciprocal kernel of $K\left(x_{0} \mid t, s\right)$ with parameter $(-1)$. Also $K^{-1}(t, s)$ is continuous and $K$ and $K^{-1}$ satisfy the two relations

$$
\begin{aligned}
K^{-1}(t, s)+K\left(x_{0} \mid t, s\right) & =-\int_{0}^{1} K^{-1}(t, u) K\left(x_{0} \mid u, s\right) d u \\
& =-\int_{0}^{1} K^{-1}(u, s) K\left(x_{0} \mid t, u\right) d u .
\end{aligned}
$$

With this $T_{1}^{-1}$ we form the transformation $T_{2}=T_{1}^{-1}$ and prove:

LEммA 11. Under the hypotheses of Theorem IV the transformation $T_{2}=T_{1}^{-1} T$ satisfies the hypotheses of Theorem III.

Proof. By (13.8) and (1.1) it is easily seen that $T_{2}$ is given by

$$
T_{2}: y(t)=x(t)+\Lambda_{2}(x \mid t)
$$

where

$$
\begin{aligned}
\Lambda_{2}(x \mid t)= & \Lambda(x \mid t)-\Lambda\left(x_{0} \mid t\right) \\
& +\int_{0}^{1} K^{-1}(t, s)\left[x(s)+\Lambda(x \mid s)-x_{0}(s)-\Lambda\left(x_{0} \mid s\right)\right] d s
\end{aligned}
$$

We next show that $\Lambda_{2}$ is of smooth variation in $S \otimes I$ and we determine its kernel. By (13.12) and (1.3)

$$
\begin{aligned}
\left.\frac{\partial}{\partial v} \Lambda_{2}(x+v y \mid t)\right]_{v=0}= & \int_{0}^{1} K(x \mid t, s) y(s) d s \\
& +\int_{0}^{1} K^{-1}(t, s)\left[y(s)+\int_{0}^{1} K(x \mid s, u) y(u) d u\right] d s \\
= & \int_{0}^{1} K_{2}(x \mid t, s) y(s) d s
\end{aligned}
$$


where

$$
K_{2}(x \mid t, s)=K(x \mid t, s)+K^{-1}(t, s)+\int_{0}^{1} K^{-1}(t, u(K(x \mid u, s) d u .
$$

Obviously $K_{2}(x \mid t, s)$ is continuous in $S \otimes I^{2}$. Hence $\Lambda_{2}$ is of smooth variation in $S \otimes I$ with kernel (13.14).

By (13.12) we see that $\Lambda_{2}\left(x_{0} \mid t\right)=0$ for $t$ in $I$, and hence $T_{2}$ satisfies condition (11.1) of Theorem III.

By (13.14) and (13.9), $K_{2}\left(x_{0} \mid t, s\right)=0$, and hence $T_{2}$ satisfies condition (11.2) of Theorem III.

By (13.14) and (11.3)

$$
\begin{aligned}
K_{2}(x \mid 0, s) & =K(x \mid 0, s)+K^{-1}(0, s)+\int_{0}^{1} K^{-1}(0, u) K(x \mid u, s) d u \\
& =K^{-1}(0, s)+\int_{0}^{1} K^{-1}(0, u) K(x \mid u, s) d u
\end{aligned}
$$

and by (13.10) and (11.3)

$$
K^{-1}(0, s)=K^{-1}(0, s)+K\left(x_{0} \mid 0, s\right)=-\int_{0}^{1} K^{-1}(u, s) K\left(x_{0} \mid 0, u\right) d u=0
$$

and hence $K_{2}$ satisfies condition (11.3).

Properties (11.4) and (11.6) for $K_{2}$ follow at once from the same properties of $K$ and the continuity of $K^{-1}(t, s)$ in $I^{2}$.

To see that $K_{2}$ satisfies condition (11.5) we first note that (13.10) together with (11.5) for $K$ ensures that $\partial K^{-1}(t, s) / \partial t$ exists and is continuous in $I^{2}$. Using this and (11.5) for $K$ and the definition (13.14) for $K_{2}$, we see that $K_{2}$ satisfies (11.5). Similarly we see that $K_{2}$ satisfies the first two parts of condition (11.7).

For the third part of condition (11.7) we first note that by (13.10)

$$
\sup _{s \in I} \underset{t \in I}{\operatorname{Var}}\left[\frac{\partial}{\partial t} K^{-1}(t, s)\right]<\infty .
$$

Using this and the fact that $K$ satisfies (the third part of) condition (11.7) we see that $K_{2}$ satisfies the third part of condition (11.7).

This concludes the proof of Lemma 11. itself.

We need a third lemma before proceeding with the proof of Theorem IV

LeMMA 12. Let $T_{1}$ and $T_{2}$ be defined as in (13.3) and (13.11). Then

$$
\Lambda(x \mid t)=\Lambda_{2}(x \mid t)+\Lambda_{1}\left[x+\Lambda_{2}(x \mid \cdot) \mid t\right]
$$

and 


$$
D(x)=D\left(x_{0}\right) D_{2}(x) .
$$

Proof. By definition (see (13.5)) $T_{1} T_{2}=T_{1}\left(T_{1}^{-1} T\right)=T$ and hence by (13.3) and (13.11), (13.15) holds.

Next, a classical result on Fredholm determinants (see, for example, $\left[2\right.$, p. 467]) states that if $f(t, s)$ and $g(t, s)$ are continuous functions on $I^{2}$ and if $\mathcal{D}_{f}$ and $\mathscr{D}_{o}$ are their Fredholm determinants formed with the parameter $(-1)$, then the product $\mathcal{D}_{f} \mathcal{D}_{\sigma}$ is the Fredholm determinant $\mathcal{D}_{\phi}$ of the kernel

$$
\phi(t, s)=f(t, s)+g(t, s)+\int_{0}^{1} f(t, u) g(u, s) d u .
$$

Applying this first to (13.9) and then to (13.14) we see that $D_{2}(x)=D(x)$ $\left[D\left(x_{0}\right)\right]^{-1}$. Hence (13.16) holds and the proof of Lemma 12 is complete.

We now proceed with the proof of Theorem IV itself. By Lemma 11 and Theorem III there exists a sphere $S_{0}$ where Theorem III applies to $T_{2}$. Let $\Gamma$ be a Wiener measurable subset of $S_{0}$ and $F(y)$ a functional which is Wiener summable on $T \Gamma$. Then by Lemma 9 and Theorem $A$ and the fact that $T=T_{1} T_{2}$ we have

$$
\begin{aligned}
& \int_{T \Gamma}^{w} F(y) d_{W} y=\left|\mathcal{D}\left(x_{0}\right)\right| \int_{T_{2} \Gamma}^{W} F\left[x+\Lambda_{1}(x \mid \cdot)\right] \\
& \cdot \exp \left\{-2 \int_{0}^{1}\left[\frac{\partial}{\partial t} \Lambda_{1}(x \mid t)\right] d x(t)-\int_{0}^{1}\left[\frac{\partial}{\partial t} \Lambda_{1}(x \mid t)\right]^{2} d t\right\} d_{w} x .
\end{aligned}
$$

By Lemma 11 (and Theorem III) this is equal to

$$
\begin{aligned}
\int_{T \Gamma}^{w} F(y) d_{W} y & =\left|D\left(x_{0}\right)\right| \int_{\Gamma}^{W} F\left\{x+\Lambda_{2}(x \mid \cdot)+\Lambda_{1}\left[x+\Lambda_{2}(x \mid \cdot) \mid \cdot\right]\right\} \\
\cdot \exp & \left\{-2 \int_{0}^{1}\left[\frac{\partial}{\partial t} \Lambda_{1}\left(x+\Lambda_{2}(x \mid \cdot) \mid t\right)\right] d_{t}\left[x(t)+\Lambda_{2}(x \mid t)\right]\right. \\
& \left.-\int_{0}^{1}\left[\frac{\partial}{\partial t} \Lambda_{1}\left(x+\Lambda_{2}(x \mid \cdot) \mid t\right)\right]^{2} d t\right\} \\
\cdot & \exp \left\{-2 \int_{0}^{1}\left[\frac{\partial}{\partial t} \Lambda_{2}(x \mid t)\right] d x(t)\right. \\
& \left.-\int_{0}^{1}\left[\frac{\partial}{\partial t} \Lambda_{2}(x \mid t)\right]^{2} d t\right\}\left|D_{2}(x)\right| d_{w} x .
\end{aligned}
$$

By Lemma 12 this yields the desired conclusion (11.8) whenever $F(y)$ is Wiener summable on $T \Gamma$. If, on the other hand, we assume that $F(y)$ is Wiener measurable on $T \Gamma$ and that the right member of (11.8) exists, then by Lemma 11 and Theorem III the right member of (13.17) exists and, by 
Lemma 9 and Theorem A, $F(y)$ is Wiener summable on TГ. This completes the proof of Theorem IV.

14. We shall now prove a transformation theorem in the large for any transformation which satisfies the hypotheses of Theorem IV locally and which is 1-to-1 in the large. More precisely, we shall prove:

THEOREM V. Let $\Gamma$ be any Wiener measurable set and assume that the transformation $T: y(t)=x(t)+\Lambda(x \mid t)$ is such that $\Lambda(x \mid t)$ is of smooth variation in a Hilbert neighborhood $S=S\left(x_{0}\right)$ of each point $x_{0}$ of $\Gamma$. Assume further that conditions (13.1), (13.2), (11.3), . . , (11.7) hold in $S\left(x_{0}\right)$ for each $x_{0}$ in $\Gamma$. Finally assume that $T$ carries $\Gamma$ in a 1-to-1 manner into a subset $T \Gamma$ of $C$. Then $T \Gamma$ is measurable and if $F(y)$ is any Wiener measurable functional on $T \Gamma$ which makes either side of (11.8) exist, the other side also exists and they are equal.

Proof. First let us recall what Theorem IV allows us to conclude. If $x_{0}$ is any point of our set $\Gamma$ then there exists a (Hilbert) neighborhood $S_{0}\left(x_{0}\right)$ contained in $S\left(x_{0}\right)$ such that the desired transformation formula holds for any Wiener measurable subset of $S_{0}\left(x_{0}\right)$. Now let $x_{1}, x_{2}, \cdots$ be a countable set of points of $\Gamma$ whose neighborhoods $S_{0}\left(x_{1}\right), S_{0}\left(x_{2}\right), \cdots$ cover $\Gamma$. (Since $C$ is a separable metric space in the Hilbert topology, any covering can be reduced to a countable covering and therefore such a set exists.) Denote by $N_{n}$ the Hilbert neighborhood $S_{0}\left(x_{n}\right)$ just described. Then $\Gamma \subset \sum N_{n}$, and if $\Gamma_{n}=N_{n} \cdot \Gamma$, we have $\Gamma=\sum \Gamma_{n}$. The desired transformation formula applies to each set $\Gamma_{n}-\sum_{k=1}^{n-1} \Gamma_{k}, n=1,2, \cdots .\left(\sum_{k=1}^{0} \Gamma_{k}\right.$ is understood to be the empty set.) Since the Wiener integral is completely additive we may sum over $n$ and thus obtain (11.8) over our given set $\Gamma$ if $F$ is summable on $T \Gamma$.

We have, accordingly, shown that (11.8) holds under the hypotheses of Theorem $\mathrm{V}$ whenever $F(y)$ is Wiener summable over $T \Gamma$. If we assume merely that $F(y)$ is Wiener measurable over $T \Gamma$ but that the right member of (11.8) exists, then the right member and hence also the left member of the corresponding equation with $\Gamma$ replaced by $\Gamma_{n}-\sum_{k=1}^{n-1} \Gamma_{k}$ exists and this is true for each $n=1,2, \ldots$. On summing over $n$ we conclude that $F(y)$ is Wiener summable on $T \Gamma$. This yields Theorem $\mathrm{V}$ in the form stated.

\section{REFERENCES}

1. R. H. Cameron and W. T. Martin, (a) Transformations of Wiener integrals under translations, Ann. of Math. vol. 45 (1944) pp.386-396; (b) Transformations of Wiener integrals under a general class of linear transformations, Trans. Amer. Math. Soc. vol. 58 (1945) pp. 184-219.

2. Gerhard Kowaleski, Einfïhrung in die Determinantentheorie, Leipzig, 1909.

3. Vito Volterra, Leçons sur les fonctions de lignes, Paris, 1913.

4. E. T. Whittaker and G. N. Watson, A course of modern analysis, New York, 1945.

5. N. Wiener, Generalized harmonic analysis, Acta Math. vol. 55 (1930) pp. 117-258.

UNIVERSITY OF MINNESOTA, MinNEAPOLIS, MinN.

MassachusetTs Institute of TeChNology, Cambridge, Mass. 